\title{
Photometric redshifts for galaxies in the Subaru Hyper Suprime-Cam and unWISE and a catalogue of identified clusters of galaxies
}

\author{
Z. L. Wen ${ }^{1,2 \star}$ and J. L. $\operatorname{Han}^{1,2,3}$ \\ 1. National Astronomical Observatories, Chinese Academy of Sciences, 20A Datun Road, Chaoyang District, Beijing 100101, China \\ 2. CAS Key Laboratory of FAST, NAOC, Chinese Academy of Sciences, Beijing 100101, China \\ 3. School of Astronomy, University of Chinese Academy of Sciences, Beijing 100049, China
}

Accepted XXX. Received YYY; in original form ZZZ

\begin{abstract}
We first present a catalogue of photometric redshifts for 14.68 million galaxies derived from the 7-band photometric data of Hyper Suprime-Cam Subaru Strategic Program and the Widefield Infrared Survey Explorer using the nearest-neighbour algorithm. The redshift uncertainty is about 0.024 for galaxies of $z \leq 0.7$, and steadily increases with redshift to about 0.11 at $z \sim 2$. From such a large data set, we identify 21,661 clusters of galaxies, among which 5537 clusters have redshifts $z>1$ and 642 clusters have $z>1.5$, significantly enlarging the high redshift sample of galaxy clusters. Cluster richness and mass are estimated, and these clusters have an equivalent mass of $M_{500} \geq 0.7 \times 10^{14} M_{\odot}$. We find that the stellar mass of the brightest cluster galaxies (BCGs) in each richness bin does not significantly evolve with redshift. The fraction of star-forming BCGs increases with redshift, but does not depend on cluster mass.
\end{abstract}

Key words: catalogues — galaxies: clusters: general — galaxies: distances and redshifts.

\section{INTRODUCTION}

According to the hierarchical scenario (Peebles 1980), clusters of galaxies were formed at knots of cosmic web and grow through accretion and merging of smaller structures (Colberg et al. 1999). Galaxy clusters therefore are one of important tracers of the large scale structure (LSS) of the Universe (Allen et al. 2011). Cosmological parameters can be constrained from the evolution of cluster number count and gas mass fraction (Allen et al. 2008; Vikhlinin et al. 2009), even constrained by a very few extremely massive clusters at high redshifts of $z>1$ (Jee et al. 2009; Hoyle et al. 2011; Brodwin et al. 2012). The properties of galaxy component in clusters show significant evolution with redshift. Cluster galaxies in the local universe are dominated by massive early-type galaxies (Rood 1969; Binggeli et al. 1987). Clusters at higher redshifts possess a higher fraction of star-forming galaxies, which is known as the Butcher-Oemler effect (Butcher \& Oemler 1978, 1984). Galaxy population in some clusters of $z>2$ is dominated by galaxies with a very high star formation rate (e.g. Wang et al. 2016; Smith et al. 2019). Specially, the brightest cluster galaxies (BCGs) are the most massive galaxies. Their properties are related to the mass and dynamical state of host clusters (Kale et al. 2015; Wen \& Han 2015; Erfanianfar et al. 2019). The stellar population in BCGs was generally formed at redshifts $z>2$

^ E-mail: zhonglue@nao.cas.cn and evolved passively afterwards (Stott et al. 2008; Whiley et al. 2008; Wen \& Han 2011).

Systematic searches for galaxy clusters from multiwavelength survey data have been carried out over a few decades since Abell (1958) and Abell et al. (1989). A large number of optical clusters have been found from the Sloan Digital Sky Survey (SDSS), most of which have a redshift of $z \lesssim 0.7$ (Koester et al. 2007; Wen et al. 2009; Hao et al. 2010; Szabo et al. 2011; Wen et al. 2012; Oguri 2014; Rykoff et al. 2014; Banerjee et al. 2018). In addition, a few thousand clusters were found from the ROentgen SATellite all sky X-ray survey (Piffaretti et al. 2011; Böhringer et al. 2013, 2017) and Planck millimeter survey (Planck Collaboration et al. 2016). At higher redshifts, some galaxy clusters have been found from deep field data covering a small area (e.g. Gladders \& Yee 2005; van Breukelen et al. 2006; Eisenhardt et al. 2008; Wen \& Han 2011). Recently, large cluster samples at $z>0.7$ were identified from the combination of optical and infrared data, for example, 1959 massive clusters of $0.7<z<1$ from the photometric data of the SDSS and Wide-field Infrared Survey Explorer (WISE) by Wen \& Han (2018), 2433 massive clusters of $0.7<z<1.5$ from the WISE data supplemented with the data of Pan-STARRS and SuperCOSMOS by Gonzalez et al. (2019). Via the Sunyaev-Zel'dovich (SZ) effect, more than one hundred massive clusters of $z>0.7$ have been previously detected from the sky surveys of cosmic microwave background by Atacama Cosmology Telescope (ACT) and South Pole Telescope (Reichardt et al. 
2013; Hilton et al. 2018). Up to now, only a few tens of clusters at $z>1.5$ have been identified or confirmed individually from optical, X-ray and SZ data (e.g. Papovich et al. 2010; Tozzi et al. 2015; Hilton et al. 2018).

Usually, optical data supplemented with infrared data are efficient to reveal high-redshift clusters. Recent public survey data provide a new opportunity for finding high-redshift galaxy clusters. Hyper Suprime-Cam Subaru Strategic Program (HSC-SSP) is a large area optical survey down to a limit of $i=26 \mathrm{mag}$, which is deep enough to detect massive galaxy of $z>2$ (Aihara et al. 2018). The WISE is an all sky survey in four mid-infrared bands (Wright et al. 2010). The recent public unWISE catalogue of the WISE reaches a limit of $\mathrm{W} 1=17.8 \mathrm{mag}$ and contains many highredshift massive galaxies (Schlafly et al. 2019).

In this paper, we first present a catalogue of photometric redshifts for galaxies in the cross-matched catalogue of the HSC-SSP and the unWISE, containing improved photometric redshifts of galaxies at $1.5<z<2$. Subsequently, a catalogue of 21,661 galaxy clusters of $0.1<z \lesssim 2$ are identified, which significantly enlarges the number of clusters at $z>1$. In Section 2, we first describe the galaxy data and the estimates of galaxy photometric redshift and stellar mass. In Section 3, we describe the cluster identification procedures and the identified galaxy clusters. In Section 4, we study the evolution of BCGs, including their stellar mass and star formation. A summary is presented in Section 5.

Throughout this paper, we assume a flat Lambda cold dark matter cosmology taking $H_{0}=70 \mathrm{~km} \mathrm{~s}^{-1} \mathrm{Mpc}^{-1}, \Omega_{m}=0.3$ and $\Omega_{\Lambda}=0.7$.

\section{PHOTOMETRIC REDSHIFTS AND STELLAR MASSES OF GALAXIES}

Based on large data set of photometric surveys, the photometric redshift of galaxies is a fundamental parameter for many further studies, such as galaxy evolution, structure formation and cluster identification. We cross-match the galaxy catalogue of the HSCSSP and the source catalogue of the unWISE to get a 7-band spectral energy distribution (SED) for the common galaxies in the two catalogues. The photometric redshift of a galaxy can thus be estimated by the comparison with a spectroscopic training sample in colour space. In addition, the stellar mass of galaxies is another important parameter in the algorithm for cluster identification, and can be estimated from the photometric data.

\subsection{Galaxy data}

The HSC-SSP ${ }^{1}$ carries out the optical photometric survey in five broad bands (grizy) and three narrow bands (NB387, NB816 and NB921) (Aihara et al. 2018). It is currently the deepest largearea optical survey, and consists of three layers of survey depth. The Wide survey aims to observe a large area of the sky covering $1400 \mathrm{deg}^{2}$ in the five broad bands, reaching a $5 \sigma$ limit of $i \sim 26$ mag for point sources. The Deep survey aims to observe four separate fields (XMM-LSS, Extended-COSMOS, ELAIS-N1 and DEEP2-F3) covering about $27 \mathrm{deg}^{2}$ in the five broad bands and the three narrow bands, reaching a limit of $i \sim 27 \mathrm{mag}$. The UltraDeep survey aims to observe two separate fields (COSMOS and SXDS) covering about $3.5 \mathrm{deg}^{2}$ in the five broad bands

\footnotetext{
1 https://hsc.mtk.nao.ac.jp/ssp/
}

and the three narrow bands, reaching a limit of $i \sim 28$ mag. The latest HSC-SSP second data release (DR2) is now publicly available for the Wide survey covering 924, 1022, 796, 905 and $924 \mathrm{deg}^{2}$ in the $g, r, i, z$ and $y$ bands, respectively, and covering about $35 \mathrm{deg}^{2}$ for the Deep+UltraDeep survey (Aihara et al. 2019). The Deep+UltraDeep field is relatively small and mostly (except the ELAIS-N1 field) overlapped by the Wide field. We here only consider the five-band photometric HSC-SSP data in the Wide field and the ELAIS-N1 field down to $i=26$ with a total sky area of $\sim 800 \mathrm{deg}^{2}$. From the HSC-SSP DR2, we get 168 million galaxies observed at least in the $r, i$ and $z$ bands with the flags of isprimary='True' (i.e. a source has no children) and i_pixelflags_saturatedcenter='False' (i.e. the source centre has no saturated pixel in the $i$ band).

The WISE survey ${ }^{2}$ observed the whole sky in four midinfrared bands (Wright et al. 2010): W1 $(3.4 \mu \mathrm{m})$, W2 $(4.6 \mu \mathrm{m})$, $\mathrm{W} 3(12 \mu \mathrm{m})$, and W4 $(22 \mu \mathrm{m})$. The WISE primary mission finished the all sky survey in 2011 and published the photometric data, known as AllWISE catalogue, in the four bands with $5 \sigma$ limits of 17.1, 15.7, 11.5, and 7.7 mag (Vega system), respectively, for point sources (Cutri \& et al. 2013). Afterwards, an asteroid-characterizing extension mission, NEOWISE, was carried out to continually survey the whole sky in the W1 and W2 bands (Mainzer et al. 2014). The unWISE catalogue ${ }^{3}$ was presented for about 2 billion sources from the unblurred coadds of five-year NEOWISE single-exposure images (Lang 2014; Schlafly et al. 2019). It reaches a limit of 0.7 mag fainter than the AllWISE catalogue and is deep enough to study massive galaxies to $z \sim 2$. We get 30 million unWISE sources with W1-band data available in the sky coverage of the HSC-SSP DR2. This number is much smaller than that of the HSC-SSP galaxies because the unWISE catalogue tends to include massive galaxies with a high mid-infrared flux. We convert the Vega magnitudes to $\mathrm{AB}$ magnitudes for the WISE sources by adding the offsets of 2.699 and 3.339 for W1 and W2 magnitudes, respectively (Jarrett et al. 2011).

We perform one-to-one matching between the HSC-SSP catalogue and the unWISE catalogue for the common galaxies, to form a catalogue of HSC-SSP $\times$ unWISE galaxies with 7-band photometric data. The unWISE sources are cross-matched with each HSCSSP galaxy using a matching radius of 2 arcsec (Bilicki et al. 2016; Shu et al. 2019). For the cases that multiple matches are found, the closest source is adopted. Then, around each matched unWISE source, the HSC-SSP galaxies are searched within the offset of 2 arcsec and the closest one is adopted for the multiple matches. Finally, we get 14.68 million HSC-SSP $\times$ unWISE galaxies.

\subsection{Photometric redshifts of galaxies}

The $4000 \AA$ break and $1.6 \mu \mathrm{m}$ bump are distinct features to determine photometric redshifts of galaxies. The 7-band photometric data of the HSC-SSP $\times$ unWISE galaxies can be used for the estimation of photometric redshifts up to $z \sim 2$. Usually, the template fitting and the empirical methods are two main approaches for the estimation of photometric redshifts. The former is applied by fitting the observed SED with a set of template SEDs in various parameter spaces to determine the most probable redshift for a galaxy (e.g. Bolzonella et al. 2000; Ilbert et al. 2006; Brammer et al. 2008;

\footnotetext{
2 http://irsa.ipac.caltech.edu/Missions/wise.html/

3 http://unwise.me/
} 


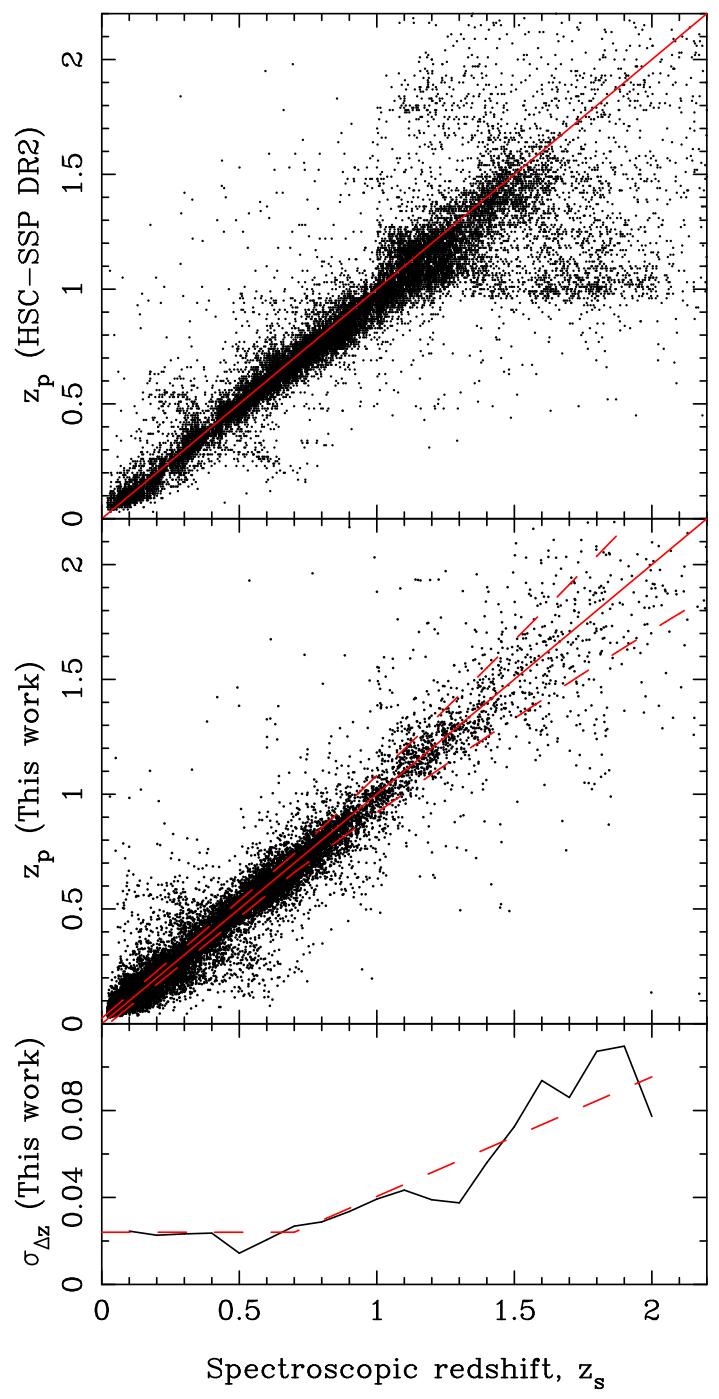

Figure 1. Comparison between photometric redshifts and spectroscopic redshifts for the HSC-SSP DR2 estimates (upper panel) and the estimates of this work (middle panel). Lower: the uncertainty of photometric redshifts of this work. The dashed lines in the middle panel indicate the deviation of $1 \sigma_{\Delta z}$, which is shown by the dashed line in the lower panel.

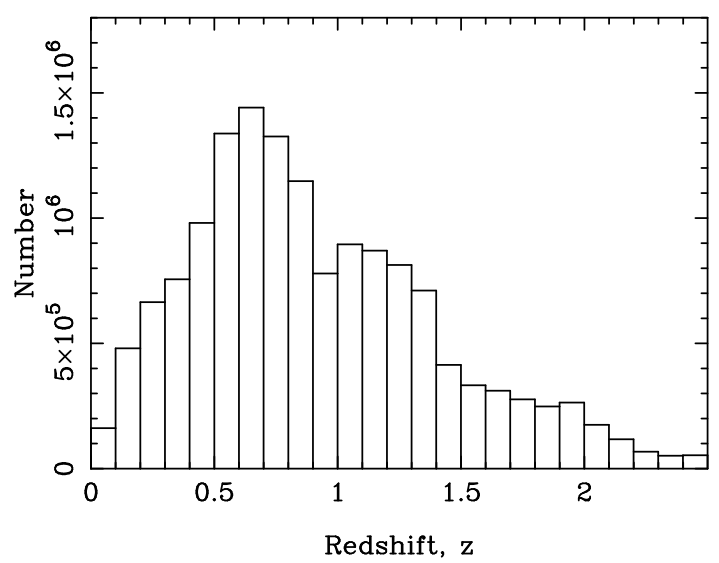

Figure 2. Distribution of photometric redshifts for 14.68 million HSCSSP $\times$ unWISE galaxies.

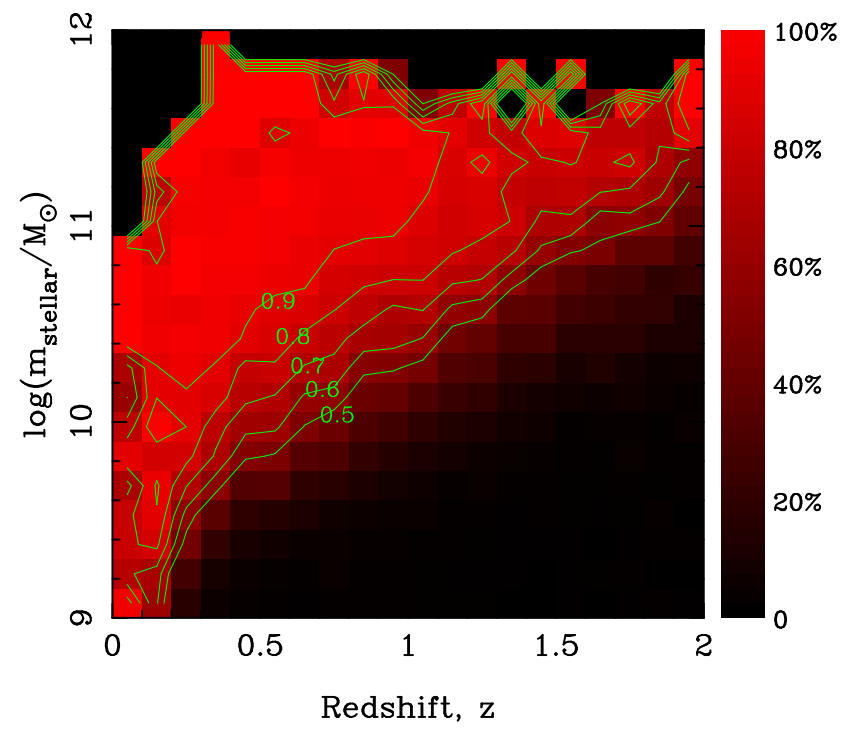

Figure 3. Completeness of galaxies in the plane of redshift and galaxy stellar mass by comparison with the COSMOS2015 data. The level of the contour decreases from $90 \%$ to $50 \%$ by an interval of $10 \%$.

Tanaka 2015). The latter is requiring a representative training sample with known spectroscopic redshifts. The photometric redshift is determined from the empirical relations between the photometric observables and the spectroscopic redshift (e.g. Collister \& Lahav 2004; Hsieh \& Yee 2014; Carrasco Kind \& Brunner 2014). Here, we estimate photometric redshifts of the HSC-SSP $\times$ unWISE galaxies by an empirical method, the nearest-neighbour algorithm (Cunha et al. 2009; Beck et al. 2016; Tanaka et al. 2018; Zou et al. 2019; Tarrío \& Zarattini 2020).

We obtain the training sample of spectroscopic redshifts from the HSC-SSP DR2, which includes the data from SDSS DR14 (Abolfathi et al. 2018), DEEP3 (Cooper et al. 2011), PRIMUS DR1 (Cool et al. 2013), VIPERS PDR1 (Garilli et al. 2014), VVDS (Le Fèvre et al. 2013), GAMA DR2 (Liske et al. 2015), WiggleZ DR1 (Drinkwater et al. 2010), zCOSMOS DR3 (Lilly et al. 2009), UDSz (Bradshaw et al. 2013; McLure et al. 2013), FMOSCOSMOS (Silverman et al. 2015; Kashino et al. 2019) and 3DHST (Skelton et al. 2014; Momcheva et al. 2016). To have enough data for training at $z>1$, we also supplement the spectroscopic redshifts with accurate photometric redshifts in the COSMOS2015 catalogue, which are based on 30-band photometry with an accuracy of 0.021 (Laigle et al. 2016). We adopt the quality flags of the redshift data following Tanaka et al. (2018). This spectroscopic sample contains 554,996 galaxies, of which 240,409 are matched with the HSC-SSP $\times$ unWISE galaxies and have magnitudes in the grizy $W 1$ bands. This obtained spectroscopic sample is biased to low redshifts due to the data from the SDSS. To reduce the bias, we randomly remove some of galaxies of $i<21.8$ (the limit of SDSS redshift survey, Prakash et al. 2016) to make that the training sample has similar colour distributions with the photometric sample following Lima et al. (2008). In the colour space $(g-r$, $r-i, i-z, z-y$ and $i-\mathrm{W} 1)$, we calculate a probability,

$p=\frac{N_{\mathrm{S}, \text { tot }} N_{\mathrm{P}}(\Delta c)}{N_{\mathrm{P}, \mathrm{tot}} N_{\mathrm{S}}(\Delta c}$.

Here, $N_{\mathrm{S} \text {, tot }}$ and $N_{\mathrm{P} \text {, tot }}$ are the total numbers of galaxies in the spectroscopic and photometric samples, respectively. $N_{\mathrm{S}}(\Delta c)$ and $N_{\mathrm{P}}(\Delta c)$ are the numbers of neighbour galaxies within the colour 
interval $\Delta c=0.05$ (for all five colours) of the galaxy from the spectroscopic and photometric samples, respectively. If $p<1$, the galaxy has the probability of $1-p$ being removed from the spectroscopic sample. We perform this with the help of a random number generator. After randomly removing the low-redshift galaxies, we adopt the remaining 208,164 galaxies as the training sample. To evaluate the accuracy of photometric redshift, we randomly select 30,000 galaxies as a test sample and take the rest much larger sample of 178,164 galaxies for training.

Galaxy colour is tightly related to redshift especially when the survey bands cover the feature of $4000 \AA$ break or $1.6 \mu \mathrm{m}$ bump. In the multidimensional colour space, the galaxies at the same locations generally have similar redshifts. For the photometric redshift of a target galaxy, we calculate the distance in the colour space ( $g-r, r-i, i-z, z-y, i-\mathrm{W} 1$ and $i-\mathrm{W} 2)$ to all the galaxies in the training sample. There are $k$ galaxies found as the nearest neighbours in the colour space. The median value of their spectroscopic redshifts is taken as the photometric redshift of the target galaxy, and the scatter of the spectroscopic redshifts of $k$ galaxies is taken as the error of estimated photometric redshift. We test various number of $k$, and find that $k=20$ can lead to the smallest scatter between the photometric redshifts and the spectroscopic redshifts for the 30,000 testing galaxies. The uncertainty of the photometric redshift in our work is defined as $\sigma_{\Delta z}=1.48 \times \operatorname{median}\left(\left|z_{p}-z_{s}\right| /\left(1+z_{s}\right)\right)$. The lower panel of Fig. 1 shows that the redshift uncertainty is about 0.024 at $z<0.7$ and steadily increases with redshift to about 0.11 at $z \sim 2$ roughly following a formula of $\sigma_{\Delta z}=0.055 z-0.0145$. We consider the photometric redshifts with a deviation larger than $3 \sigma_{\Delta z}$ or larger than 0.15 as outliers, which is about $6 \%$ for our photometric redshift estimates.

The HSC-SSP has already published photometric redshifts ${ }^{4}$ for all HSC-SSP galaxies based on the five band (grizy) data. The photometric redshift catalogue of DR1 (Tanaka et al. 2018) contains six estimates based on one template fitting method (Mizuki; Tanaka 2015) and five neural network or machine learning methods, i.e. Direct Empirical Photometric code (DEmP; Hsieh \& Yee 2014), Extended Photometric redshift, Flexible Regression over Associated Neighbours with Kernel dEnsity estimatioN for Redshifts, MLZ (Carrasco Kind \& Brunner 2014) and Nearest Neighbours Photometric Redshift (Cunha et al. 2009), respectively. The photometric redshifts of the HSC-SSP DR2 galaxies are provided within $z<5$ based on the DEmP and Mizuki methods (Nishizawa et al. 2020). The redshift estimates of the HSC-SSP have an uncertainty of about 0.05 and an outlier rate of about $15 \%$ for the galaxies down to $i=25$. In Fig. 1, we compare both photometric redshifts in the HSC-SSP DR2 (Mizuki estimate) and in this work with spectroscopic redshifts. The HSC-SSP DR2 redshift has a larger uncertainty at $1.4<z<2$ mainly because the $4000 \AA$ A break moves out of the $y$ band, which can be improved with the help of the unWISE data.

We then take all 208,164 galaxies as the training sample, and obtain photometric redshifts for 14.68 million HSC-SSP $\times$ unWISE galaxies $^{5}$ using the nearest-neighbour algorithm. The redshift distribution of the HSC-SSP $\times$ unWISE galaxies has a peak at $z \sim$ 0.7 and extends to $z>2$, as shown in Fig. 2. The deeper COSMOS2015 data can be used to estimate the completeness of the HSC-SSP $\times$ unWISE data. The completeness is defined to be

\footnotetext{
4 https://hsc-release.mtk.nao.ac.jp/doc/index.php/photometric-redshifts/

5 All data are available at http://zmtt.bao.ac.cn/galaxy_clusters/
}

the percentage calculated from the number of galaxies in HSCSSP $\times$ unWISE catalogue over the number of galaxies in the COSMOS2015 catalogue. As shown in Fig. 3, the completeness of galaxies depends on stellar mass and redshift. For the galaxies with a stellar mass of $m_{\text {stellar }} \sim 10^{11} M_{\odot}$, the completeness decreases from $>90 \%$ at $z<1$ to $\sim 70 \%$ at $z \sim 1.5$. For the galaxies with a stellar mass of $m_{\text {stellar }} \sim 10^{10.5} M_{\odot}$, the completeness drops from $\sim 90 \%$ at $z<0.5$ to $\sim 70 \%$ at $z \sim 1$. The completeness may vary across the sky because the unWISE catalogue is not homogeneous and is relatively shallow in the COSMOS field (Schlafly et al. 2019). The completeness of galaxies in other regions, however, currently cannot be evaluated.

\subsection{Stellar masses of galaxies}

The stellar masses $\left(m_{\text {stellar }}\right)$ of galaxies have a tight correlation with infrared luminosities (Bell \& de Jong 2001; Drory et al. 2004; Rigopoulou et al. 2006; Li et al. 2007). We here derive the stellar mass based on the W1-band luminosity of galaxies. First we convert the W1-band magnitude to a luminosity by

$\log \left(L_{\mathrm{W} 1} / L^{*}\right)=-0.4\left(\mathrm{~W} 1-\mathrm{W} 1^{*}\right)$,

where $\mathrm{W} 1^{*}$ is the reference characteristic magnitude from a passive population synthesis model of Bruzual \& Charlot (2003), normalized to $\mathrm{W} 1^{*}=16.45$ at $z=0.1$ (Mancone et al. 2010; Gonzalez et al. 2019). In the evolution model, we assume that the stellar population was formed at a redshift of $z_{f}=3$, and adopt the stellar evolution tracks of Padova 1994 (Girardi et al. 1996), the 'Basel3.1' stellar spectral library (Westera et al. 2002), and the initial mass function of Chabrier (2003) and the Solar metallicity. The $L^{*}$ is the corresponding characteristic luminosity.

To calibrate the relation between galaxy stellar mass and infrared luminosity, we use the stellar mass data in the COSMOS2015 catalogue, which are based on 30-band photometry for half million galaxies to a limit of $K_{s}=24.7$ (Laigle et al. 2016). Cross-matching the COSMOS2015 catalogue with the HSC-SSP $\times$ unWISE catalogue gives 33,879 common galaxies at $z<2.5$. We show the correlation between $L_{\mathrm{W} 1}$ and $m_{\text {stellar }}$ for the 33,879 galaxies (the upper panel of Fig. 4) and fit the relation to a power law for the whole sample,

$\log \left(m_{\text {stellar }} / M_{\odot}\right)=\gamma \log \left(L_{\mathrm{W} 1} / L^{*}\right)+f$.

The best fitting gives the slope of $\gamma=1.13 \pm 0.01$ and the intercept of $f=10.63 \pm 0.01$. The slope is consistent with that derived by Wen et al. (2013). The scatter of stellar masses, $1.48 \times \operatorname{median}\left(\Delta m_{\text {stellar }}\right)$, is about $0.32 \mathrm{dex}$ as estimated by Eq. (3). Considering redshift dependence from possible physical evolution of the luminosity-stellar relation or any bias of the quoted reference $\mathrm{W} 1^{*}$ (Wen \& Han 2018) and the colour dependence (Bell \& de Jong 2001; Wen et al. 2013; Cluver et al. 2014), we divide the galaxy sample into small redshift bins, and fit the $L_{\mathrm{W} 1}-m_{\text {stellar }}$ relation to the power law in Eq. 3 but with the intercept $f$ in the form of

$f=a(z)+b(z)(r-Z)$.

The values of $a(z)$ and $b(z)$ are used to diminish the redshift dependence, and are derived within each redshift bin (see middle panels of Fig. 4). The $(r-Z)$ in Eq. 4 is the colour index ( $Z$ is for the filter band to avoid confusion with redshift in this equation). After such corrections, the accuracy of estimated stellar mass is improved from 0.32 dex to 0.21 dex (see the lower panel of 

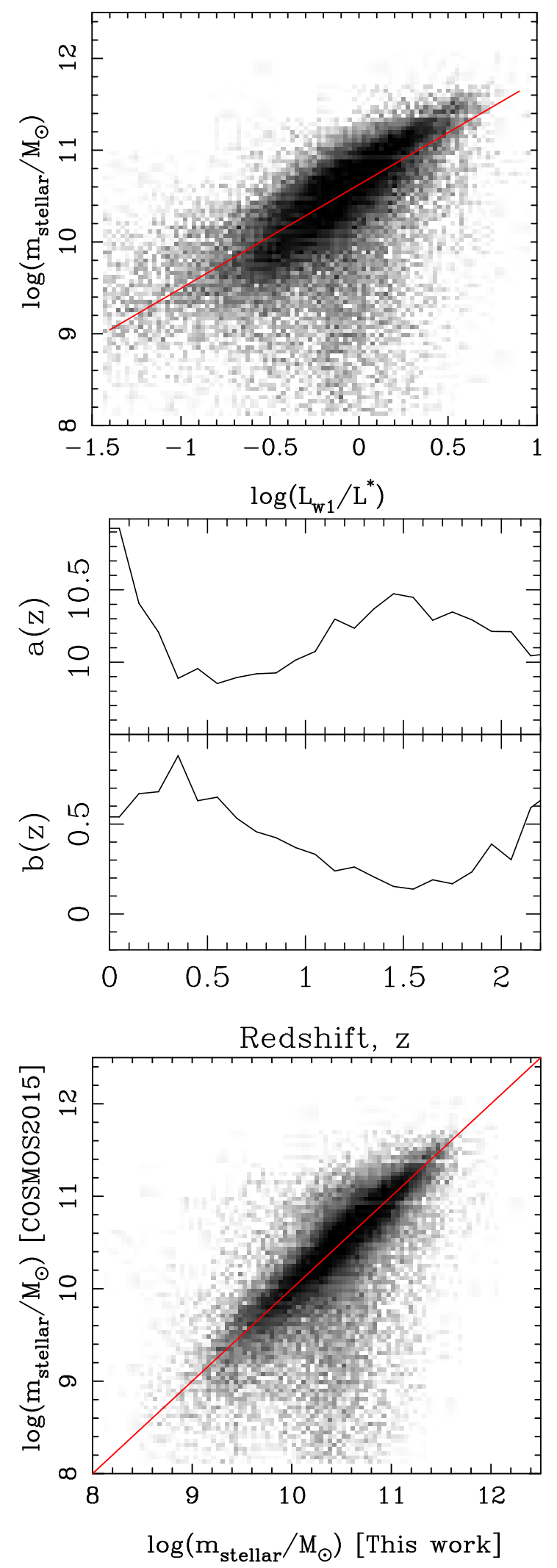

Figure 4. Upper: correlation between galaxy stellar mass and W1-band luminosity. Middle: The slope and intercept of the relation between stellar mass and galaxy colour as a function of redshift, which are used for improvement of the stellar mass-luminosity relation. Lower: comparison between the stellar mass in the COSMOS2015 catalogue and the estimate in this work.

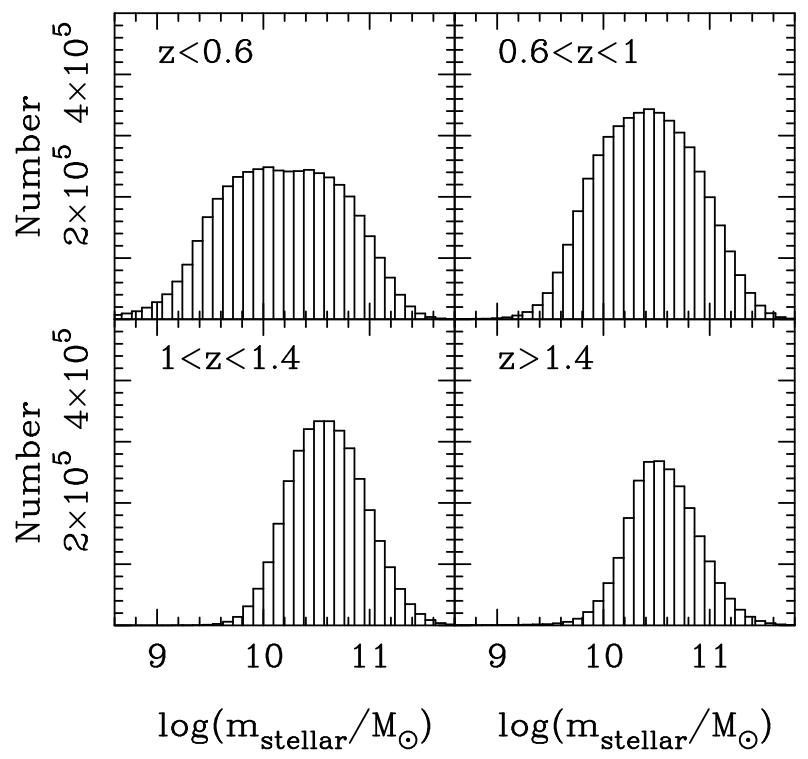

Figure 5. Distribution of stellar masses of HSC-SSP $\times$ unWISE galaxies at different redshift ranges.

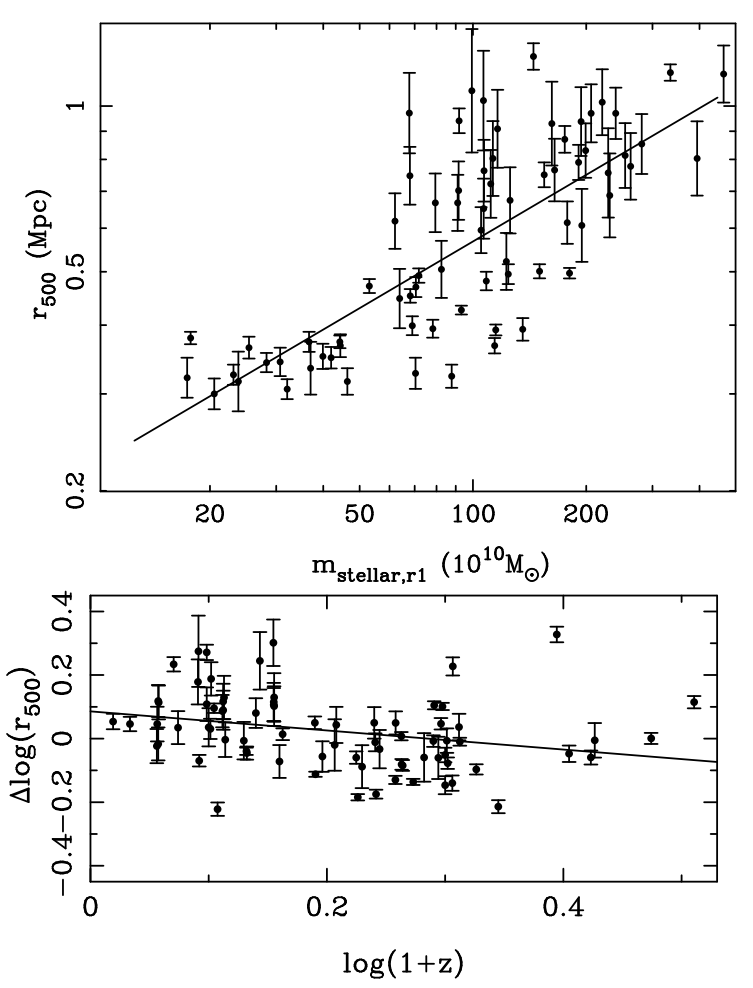

Figure 6. Upper: the scaling relation between cluster radius, $r_{500}$, and the total stellar mass, $m_{\text {stellar,r1 }}$, derived from HSC-SSP $\times$ unWISE data for the test sample of 76 clusters from literature. The solid line is the best fit to the data. Lower: the deviation of $r_{500}$ from the $r_{500}-m_{\text {stellar,r1 }}$ relation against $\log (1+z)$.

Fig. 4). We therefore calculate the stellar masses for 14.68 million HSC-SSP $\times$ unWISE galaxies. As shown in Fig. 5, the HSC$\mathrm{SSP} \times$ unWISE galaxies are more massive at higher redshifts in general. 

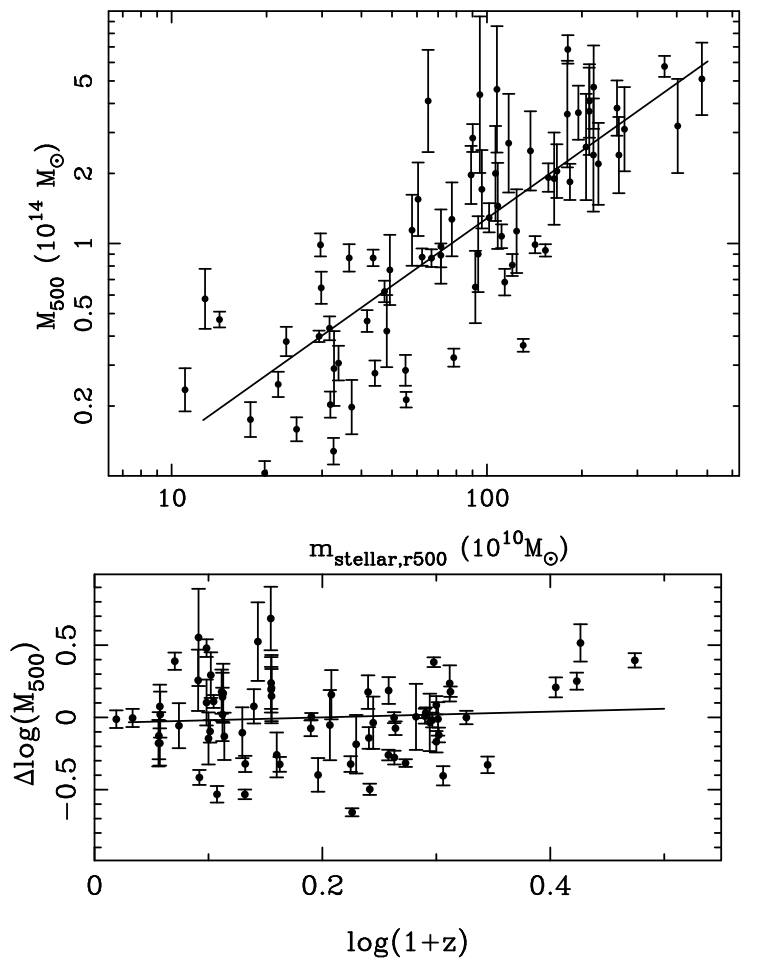

Figure 7. Similar to Fig. 6, but for the $M_{500}-m_{\text {stellar,r500 }}$ relation determined for a test sample of clusters.

\section{CLUSTERS OF GALAXIES IDENTIFIED FROM THE PHOTOMETRIC REDSHIFT CATALOGUE}

With the improved photometric redshifts of 14.68 million HSCSSP $\times$ unWISE galaxies, we can identify clusters of galaxies from the three-dimensional distribution of galaxies, following the steps in our previous papers (Wen et al. 2009, 2012; Wen \& Han 2015). Here, we first verify the scaling relations for the radius cluster and mass from observables for a test sample of clusters, which will be used during the identification of galaxy clusters.

\subsection{The scaling relations for cluster radius and mass}

Mass and radius are fundamental parameters of a cluster. The common used radius, $r_{500}$, is the radius within which the mean density of a cluster is 500 times of the critical density of the universe, and $M_{500}$ is the cluster mass within $r_{500}$. Determining the scaling relations with observables of member galaxies is a prior step in our cluster identification algorithm. As done in our previous work, we used the total luminosity of member galaxies as the cluster mass proxy for the SDSS clusters (Wen \& Han 2015). Here, we have the stellar masses of galaxies derived already. We re-calibrate the scaling relation between the cluster mass (and radius) and the total stellar mass in clusters.

Following our previous papers (Wen et al. 2012; Wen \& Han 2015), we use the clusters with known $M_{500}$ (and $r_{500}$ ) as the test sample to get the scaling relations. The HSC-SSP $\times$ unWISE data cover the fields of the deep X-ray surveys, e.g. the Chandra Allwavelength Extended Groth Strip International Survey (Laird et al. 2009), XMM-LSS survey (Pierre et al. 2004) and XMM XXL survey (Pierre et al. 2016). We find the cluster masses of $M_{500}$ for 76 known clusters in the HSC-SSP $\times$ unWISE data (Erfanianfar et al. 2013; Pacaud et al. 2016; Adami et al. 2018; Willis et al. 2018).
The locations of BCGs are taken as the centres of these clusters. We discriminate the member galaxy candidates with a stellar mass of $m_{\text {stellar }} \geq 5 \times 10^{9} M_{\odot}$ in the HSC-SSP $\times$ unWISE data if they are within the redshift slice of $z \pm \Delta z$. Here, $\Delta z$ is adopted in a simple form of

$\Delta z= \begin{cases}0.04(1+z) & \text { for } z \leq 0.7 \\ 0.15 z-0.037 & \text { for } z>0.7\end{cases}$

which is about $1.5 \sigma_{\Delta z}$ at $z<1.4$. Within this photometric redshift slice, most member galaxies of a cluster can be included with a relatively small contamination from field galaxies that will be subtracted on average later (Wen et al. 2009).

To get the scaling relation for $r_{500}$, the total stellar mass is calculated by summing the stellar masses of so-recognized member galaxy candidates within a temporary radius, with a local background subtracted. Instead of using a radius of $1 \mathrm{Mpc}$ as in our previous papers (Wen et al. 2012; Wen \& Han 2015), we adopt the radius as

$r_{1}=1.0 E(z)^{-1 / 3} \mathrm{Mpc}$,

where $E(z)=\sqrt{\Omega_{\Lambda}+\Omega_{m}(1+z)^{3}}$, because the radius of a $M_{500}$-fixed cluster changes with redshift by a factor of $E(z)^{-1 / 3}$. The local background of the stellar mass is estimated from an annulus of projected distance between 2 and $4 \mathrm{Mpc}$ from the BCG within the same redshift slice. We find that the $r_{500}$ is well related to the total stellar mass $m_{\text {stellar,r1 }}$ within $r_{1}$ following a power law (upper panel of Fig. 6),

$\log r_{500}=\alpha \log m_{\text {stellar,r1 }}-\beta$,

where $r_{500}$ is in units of Mpc and $m_{\text {stellar,r1 }}$ is in units of $10^{10} \mathrm{M}_{\odot}$. The direct fitting by this small test sample of X-ray flux-limited clusters usually suffers from the Malmquist bias (Mantz et al. 2010; Oguri et al. 2018). We therefore prefer to fixing the slope of this scaling relation according to the result of Wen \& Han (2015) obtained from a much large sample of clusters, which is 0.45 for the cluster radius-luminosity relation in the SDSS $r$ band. We assume that the slopes of the stellar mass-luminosity relation in the $r$ and W1 bands are consistent (Bernardi et al. 2010). Considering such an additional slope of 1.13 (Eq. 3), we get the slope of $\alpha=0.45 / 1.13=0.40$ and find the best fitting of $\beta=$ $1.05 \pm 0.02$. The deviations of $r_{500}$ from the radius-stellar mass relation, $\Delta \log r_{500}=\log r_{500}-\left(0.40 \log m_{\text {stellar,r1 }}-1.05\right)$, have a negative redshift dependence in the form of (lower panel of Fig. 6)

$\Delta \log r_{500}=-0.33 \pm 0.12 \log (1+z)+(0.09 \pm 0.03)$.

Combination of equations (7) and (8) indicates that $r_{500}$ can be derived from the total stellar mass and redshift by

$$
\begin{aligned}
\log r_{500}= & 0.40 \log m_{\text {stellar, } \mathrm{r} 1}-(0.96 \pm 0.03) \\
& +(0.33 \pm 0.12) \log (1+z) .
\end{aligned}
$$

To get the scaling relation for $M_{500}$, we calculate the to-

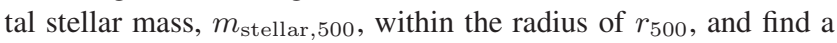
reasonable correlation between $M_{500}$ (in units of $10^{14} M_{\odot}$ ) and

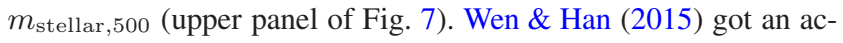
curate slope of 1.08 for the cluster mass-luminosity relation in the $r$ band. We assume a power law for the cluster mass-stellar mass relation and fix the slope to be $1.08 / 1.13=0.96$ after considering the conversion with an additional slope between luminosity and stellar mass. The best fit we obtain is

$\log M_{500}=0.96 \log m_{\text {stellar, } 500}-(1.82 \pm 0.02)$.

The deviations of $M_{500}$ from the cluster mass-stellar mass relation, 
Table 1. The 21,661 clusters of galaxies identified from the HSC-SSP $\times$ unWISE data.

\begin{tabular}{ccccccccccccc}
\hline $\begin{array}{c}\text { Cluster ID } \\
(1)\end{array}$ & $\begin{array}{c}\text { Name } \\
(2)\end{array}$ & $\begin{array}{c}\text { R.A. } \\
(3)\end{array}$ & $\begin{array}{c}\text { Dec. } \\
(4)\end{array}$ & $\begin{array}{c}z_{\mathrm{cl}} \\
(5)\end{array}$ & $\begin{array}{c}i_{\mathrm{BCG}} \\
(6)\end{array}$ & $\begin{array}{c}\text { W1 } 1_{\mathrm{BCG}} \\
(7)\end{array}$ & $\begin{array}{c}\text { SNR } \\
(8)\end{array}$ & $\begin{array}{c}r_{500} \\
(9)\end{array}$ & $\begin{array}{c}\lambda_{500} \\
(10)\end{array}$ & $\begin{array}{c}M_{500} \\
(11)\end{array}$ & $\begin{array}{c}N_{\text {gal }} \\
(12)\end{array}$ & $\begin{array}{c}\text { Other catalogues } \\
(13)\end{array}$ \\
\hline 1 & WH J000000.5+021911 & 0.00201 & 2.31980 & 0.4297 & 18.791 & 17.964 & 6.73 & 0.560 & 21.16 & 0.96 & 14 & \\
2 & WH J000004.2+021941 & 0.01741 & 2.32800 & 0.6165 & 19.557 & 17.721 & 6.02 & 0.656 & 30.51 & 1.36 & 17 & WHL \\
3 & WH J000007.9+005153 & 0.03273 & 0.86473 & 0.9170 & 21.493 & 18.930 & 5.17 & 0.491 & 20.54 & 0.93 & 10 & \\
4 & WH J000009.0+021817 & 0.03743 & 2.30465 & 0.7390 & 19.643 & 17.888 & 6.32 & 0.610 & 28.34 & 1.27 & 15 & \\
5 & WH J000011.9-013226 & 0.04941 & -1.54056 & 1.3002 & 23.644 & 19.698 & 5.20 & 0.500 & 26.45 & 1.19 & 9 & \\
6 & WH J000014.7+005259 & 0.06120 & 0.88297 & 0.6746 & 19.176 & 17.811 & 5.47 & 0.593 & 23.12 & 1.05 & 12 & \\
7 & WH J000015.0-003444 & 0.06253 & -0.57879 & 0.7652 & 20.286 & 17.729 & 7.53 & 0.610 & 32.70 & 1.46 & 12 & WHL \\
8 & WH J000016.1+022955 & 0.06711 & 2.49857 & 0.4365 & 18.671 & 17.910 & 5.53 & 0.625 & 17.41 & 0.80 & 13 & WHL \\
9 & WH J000017.4-004841 & 0.07260 & -0.81148 & 0.6165 & 19.392 & 17.836 & 9.24 & 0.671 & 40.08 & 1.77 & 23 & WH \\
10 & WH J000020.3+014340 & 0.08467 & 1.72774 & 0.8528 & 21.824 & 19.052 & 5.95 & 0.630 & 30.35 & 1.36 & 16 & \\
\hline
\end{tabular}

Note. Column 1: Cluster ID; Column 2: Cluster name with J2000 coordinates of cluster; Column 3 and 4: Right Ascension (R.A. J2000) and Declination (Dec. J2000) of cluster BCG (in degree); Column 5: redshift of the cluster; Column 6-7: BCG magnitudes (AB system) in $i$ and W1 bands, respectively; Column 8: signal-to-noise ratio for cluster detection; Column 9: cluster radius, $r_{500}$, in Mpc; Column 10: cluster richness. Column 11: derived cluster mass, in units of $10^{14} M_{\odot}$; Column 12: number of member galaxy candidates within $r_{500}$; Column 13: Reference notes for 6047 previously known clusters: WH11 (Wen \& Han 2011), WHL (Wen et al. 2012; Wen \& Han 2015), CAMIRA14 (Oguri 2014), CAMIRA18 (Oguri et al. 2018), WH18 (Wen \& Han 2018), XXL (Adami et al. 2018), ACT (Hilton et al. 2018), MaDCoWS (Gonzalez et al. 2019).

(This table is available in its entirety in a machine-readable form.)

$\Delta \log M_{500}=\log M_{500}-\left(0.96 \log m_{\text {stellar }, 500}-1.82\right)$, have a very weak dependence on redshift in the form of (lower panel of Fig. 7)

$\Delta \log M_{500}=(0.20 \pm 0.31) \log (1+z)-(0.04 \pm 0.07)$.

Combination of equations (10) and (11) indicates that $M_{500}$ can be derived from the total stellar mass and redshift by

$$
\begin{aligned}
\log M_{500}= & 0.96 \log m_{\text {stellar }, 500}-(1.86 \pm 0.07) \\
& +(0.20 \pm 0.31) \log (1+z) .
\end{aligned}
$$

Furthermore, we define the richness $\lambda_{500}$ as a mass proxy by

$\lambda_{500}=m_{\text {stellar }, 500}(1+z)^{0.21} / m_{\text {stellar }}^{*}$,

where $m_{\text {stellar }}^{*} \sim 4 \times 10^{10} M_{\odot}$ is the mean stellar mass of a galaxy with the luminosity of $L^{*}$ at $z \sim 0.1$. The cluster mass, $M_{500}$, is related to the richness, $\lambda_{500}$, by

$\log M_{500}=0.96 \log \lambda_{500}-(1.29 \pm 0.02)$,

which is redshift independent and generally in agreement with that in our previous work (Wen \& Han 2015).

\subsection{Identification of galaxy clusters}

In optical/infrared data, galaxy clusters are indicated by a high overdensity of member galaxies. Since galaxy population in clusters is dominated by massive galaxies with a high stellar mass, the total stellar mass of member galaxies is not seriously affected by the incompleteness of low-mass galaxies. Using the estimated photometric redshifts and stellar masses of galaxies, we can identify galaxy clusters from the HSC-SSP $\times$ unWISE galaxies following the approach in our previous papers (Wen et al. 2009, 2012, 2018), which includes following four steps:

1. Estimate redshifts for cluster candidates. Clusters usually contain BCGs with a high stellar mass (Lin et al. 2017). We temporarily assume that each massive galaxy of $m_{\text {stellar }} \geq 10^{11} M_{\odot}$ is the BCG of a cluster candidate at $z$. The member galaxy candidates with a stellar mass of $m_{\text {stellar }} \geq 5 \times 10^{9} M_{\odot}$ are searched from

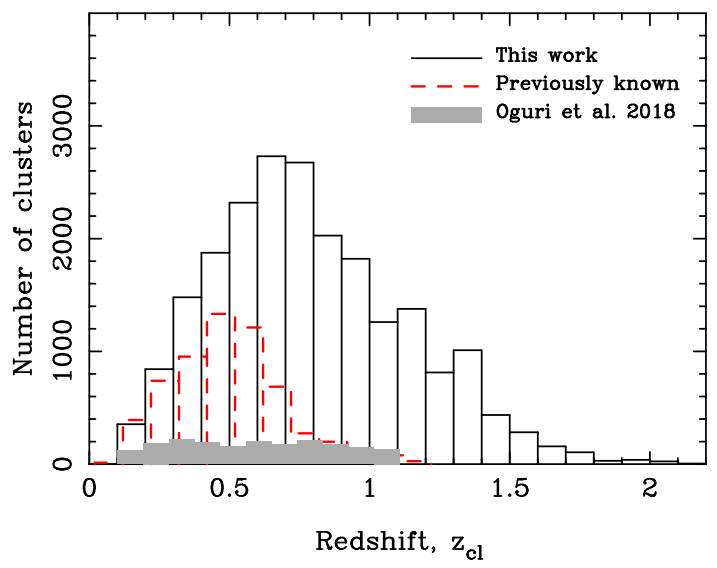

Figure 8. Redshift distribution of the 21,661 clusters identified from the HSC-SSP $\times$ unWISE data. The dashed histogram indicates the distribution of 6047 clusters previously known. The grey is for the CAMIRA clusters from HSC Wide S16A data release (Oguri et al. 2018).

the galaxies within the photometric redshift slice of $z \pm \Delta z$ and the radius of $r_{1}$. The cluster redshift is taken to be the median value of the photometric redshifts of these member galaxy candidates.

2. Calculate a signal-to-noise ratio of the overdensity for cluster candidates. For each cluster candidate, we get the sum of stellar mass of member galaxy candidates within a projected radius of $0.5 \mathrm{Mpc}$ from the $\mathrm{BCG}, m_{\text {stellar,0.5. We also esti- }}$ mate a local "background", $\left\langle m_{\text {stellar, } 0.5}\right\rangle$, and a "fluctuation", $\sigma_{m_{\text {stellar,0.5 }}}$, of the stellar mass within the same redshift slice (Wen et al. 2018; Wen \& Han 2018). The "signal-to-noise ratio" of an overdensity region is defined as SNR $=\left(m_{\text {stellar }, 0.5}-\right.$

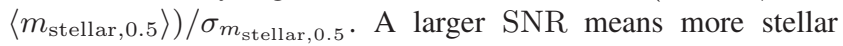
mass of galaxies concentrated on the BCG candidates, which indicates a higher likelihood of true clusters.

3. Derive the radius and richness of cluster candidates based on the scaling relations obtained in Section 3.1. We first calculate the total stellar mass, $m_{\text {stellar,r1 }}$, within the radius of $r_{1}$ from the 

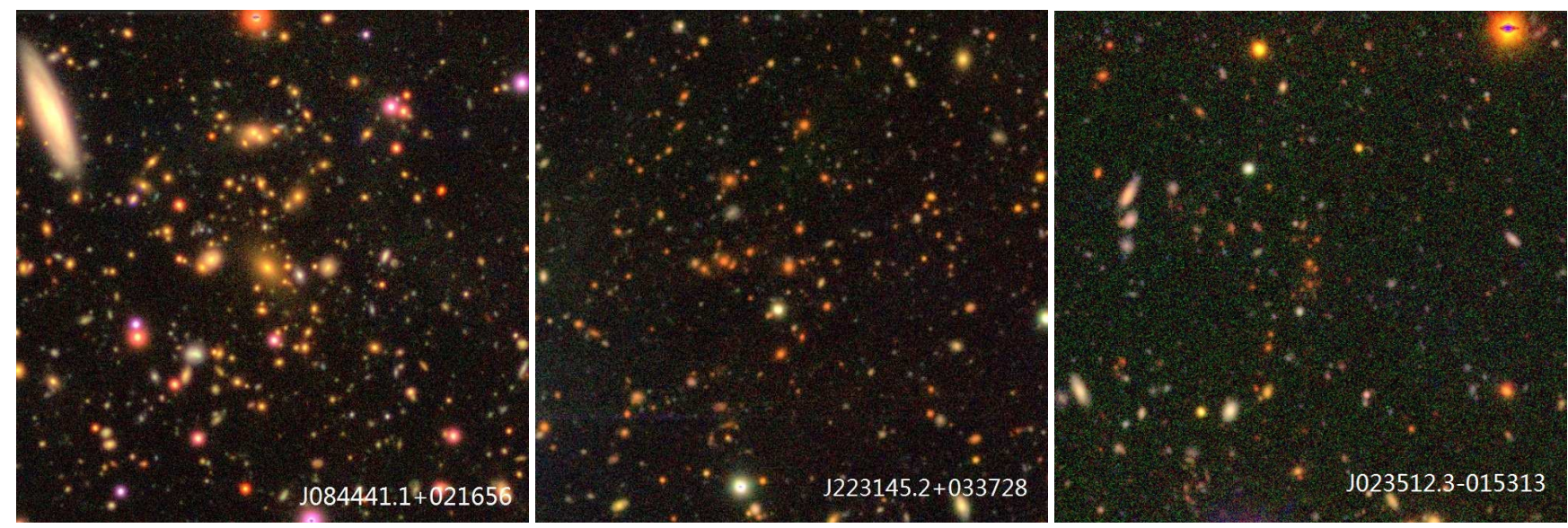

Figure 9. HSC-SSP composite ( $r i z$ ) colour images of three clusters at $z_{\mathrm{cl}}=0.6579$ (left), 0.9986 (middle) and 1.4594 (right), respectively, identified from the HSC-SSP $\times$ unWISE data. The images have a scale of $1 \mathrm{Mpc} \times 1 \mathrm{Mpc}$.

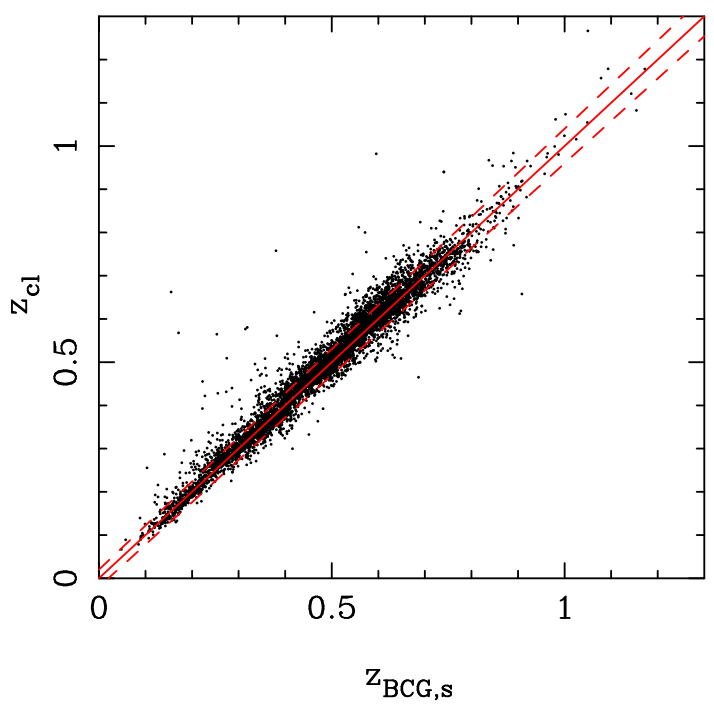

Figure 10. Comparison between the estimated cluster redshifts with spectroscopic redshifts of 6310 BCGs. The red dashed lines indicate the accuracy as being $0.022(1+z)$.

HSC-SSP $\times$ unWISE data, so that $r_{500}$ is derived using the Eq. (9). Then, we calculate the total stellar mass, $m_{\text {stellar,500, within the }}$ estimated radius $r_{500}$. The cluster richness, $\lambda_{500}$, is derived using the Eq. (13).

4. Clean the entries. A rich cluster usually contains several massive galaxies with $m_{\text {stellar }} \geq 10^{11} M_{\odot}$, so that a cluster can be repeatedly identified in the above procedures. The repeated entries have to be merged into one cluster if they have a redshift difference smaller than $1.5 \Delta z$ and a projected distance smaller than $1.5 r_{500}$. The entry with the largest richness is then adopted for such a multiidentified cluster.

After these steps, we select only the clusters with a high overdensity of SNR $\geq 5$ and a richness of $\lambda_{500} \geq 15$. The masses of so identified clusters have $M_{500} \geq 0.7 \times 10^{14} M_{\odot}$ according to the Eq. (14). To avoid false detections with very few member galaxies at high redshifts, we set the threshold for the number of member galaxy candidates within $r_{500}$ as being $N_{\text {gal }} \geq 6$. The clusters of $z_{\mathrm{cl}}<0.1$ are also discarded because bright HSC-SSP galaxies are possibly saturated and are not included in the HSC-SSP $\times$ unWISE data. Finally, we get 21,661 clusters from the HSC-SSP $\times$ unWISE data, as listed in Table 1, among which 6047 clusters are previously known (Wen \& Han 2011; Wen et al. 2012; Wen \& Han 2015; Oguri 2014; Oguri et al. 2018; Wen \& Han 2018; Adami et al. 2018; Hilton et al. 2018; Gonzalez et al. 2019). Fig. 8 shows that the identified clusters have a redshift in the range of $0.1<z_{\mathrm{cl}} \lesssim 2$ with a peak at $z_{\mathrm{cl}} \sim 0.7$. There are 5537 clusters at $z_{\mathrm{cl}} \geq 1$ and 642 clusters at $z_{\mathrm{cl}} \geq 1.5$. About $90 \%$ of clusters are newly identified at $z_{\mathrm{cl}}>0.7$. In Fig. 9, we show three identified clusters from the HSC-SSP $\times$ unWISE data at $z_{\mathrm{cl}}=0.6579,0.9986$ and 1.4594 , respectively. The cluster at $z_{\mathrm{cl}}=0.6579$ shows many member galaxies and has the BCG with magnitudes of $i=19.260$ and $\mathrm{W} 1=17.659$. The cluster at $z_{\mathrm{cl}}=1.4594$ has the BCG with magnitudes of $i=22.757$ and $\mathrm{W} 1=18.922$. Considering the data limit of the unWISE, we can see the concentration of the member galaxies 1.5 mag fainter than the BCG in this cluster centre. By comparing with available spectroscopic redshifts of 6310 BCGs, we find that the estimated cluster redshift has an accuracy of $\left(z_{\mathrm{cl}}-z_{\mathrm{BCG}, \mathrm{s}}\right) /\left(1+z_{\mathrm{BCG}, \mathrm{s}}\right)$, which is about 0.022 (see Fig. 10). The member candidates of 372,974 galaxies within $r_{500}$ for the 21,661 clusters are listed in Table 2 . 


\begin{tabular}{|c|c|c|c|c|c|c|c|c|c|c|c|c|c|c|c|c|c|c|c|}
\hline $\begin{array}{l}\text { CluID } \\
\text { (1) }\end{array}$ & $\begin{array}{l}\text { R.A. } \\
\text { (2) }\end{array}$ & $\begin{array}{l}\text { Dec. } \\
\text { (3) }\end{array}$ & $\begin{array}{c}z \\
(4)\end{array}$ & $\begin{array}{c}g \\
(5)\end{array}$ & $\begin{array}{l}\operatorname{err}_{g} \\
(6)\end{array}$ & $\begin{array}{c}r \\
(7)\end{array}$ & $\begin{array}{l}\operatorname{err}_{r} \\
(8)\end{array}$ & $\begin{array}{c}i \\
(9)\end{array}$ & $\begin{array}{l}\operatorname{err}_{i} \\
(10)\end{array}$ & $\begin{array}{c}Z \\
(11)\end{array}$ & $\begin{array}{c}\operatorname{err}_{Z} \\
(12)\end{array}$ & $\begin{array}{c}y \\
(13)\end{array}$ & $\begin{array}{l}\operatorname{err}_{y} \\
\text { (14) }\end{array}$ & $\begin{array}{l}\text { W1 } \\
\text { (15) }\end{array}$ & $\begin{array}{l}\text { errW1 } \\
\text { (16) }\end{array}$ & $\begin{array}{l}\text { W2 } \\
\text { (17) }\end{array}$ & $\begin{array}{l}\text { errW2 } \\
\text { (18) }\end{array}$ & $\begin{array}{c}\log \left(m_{\text {stellar }}\right) \\
(19)\end{array}$ & $\begin{array}{c}r_{p} \\
(20)\end{array}$ \\
\hline 1 & 0.00009 & 2.30655 & 0.4775 & 22.898 & 0.017 & 21.751 & 0.006 & 21.151 & 0.004 & 20.828 & 0.007 & 20.584 & 0.012 & 19.885 & 0.060 & 20.299 & 0.183 & 10.163 & 0.270 \\
\hline 1 & 0.00956 & 2.30905 & 0.4423 & 22.835 & 0.016 & 21.252 & 0.005 & 20.508 & 0.003 & 20.191 & 0.004 & 19.997 & 0.008 & 19.630 & 0.048 & 99.000 & 99.000 & 10.398 & 0.265 \\
\hline 1 & 0.01818 & 2.32395 & 0.4118 & 22.593 & 0.011 & 20.854 & 0.003 & 20.073 & 0.002 & 19.727 & 0.003 & 19.546 & 0.004 & 19.116 & 0.031 & 19.564 & 0.098 & 10.732 & 0.337 \\
\hline 1 & 0.01162 & 2.29570 & 0.4373 & 23.834 & 0.032 & 22.331 & 0.009 & 21.638 & 0.007 & 21.394 & 0.013 & 21.178 & 0.024 & 20.819 & 0.137 & 99.000 & 99.000 & 9.793 & 0.523 \\
\hline 1 & 0.01247 & 2.30269 & 0.4400 & 21.936 & 0.010 & 20.238 & 0.003 & 19.431 & 0.001 & 19.061 & 0.002 & 18.861 & 0.003 & 18.442 & 0.018 & 19.055 & 0.063 & 11.011 & 0.405 \\
\hline 1 & 0.02738 & 2.31081 & 0.3919 & 21.991 & 0.007 & 20.414 & 0.002 & 19.724 & 0.001 & 19.416 & 0.002 & 19.250 & 0.004 & 18.864 & 0.025 & 19.457 & 0.089 & 10.784 & 0.543 \\
\hline 1 & 0.01404 & 2.31318 & 0.4292 & 22.163 & 0.010 & 20.452 & 0.003 & 19.692 & 0.001 & 19.339 & 0.002 & 19.148 & 0.004 & 18.036 & 0.013 & 18.466 & 0.038 & 11.172 & 0.277 \\
\hline 1 & 0.00201 & 2.31980 & 0.4385 & 21.167 & 0.007 & 19.597 & 0.002 & 18.791 & 0.001 & 18.372 & 0.002 & 18.121 & 0.003 & 17.964 & 0.013 & 18.254 & 0.032 & 11.259 & 0.000 \\
\hline 1 & 0.02213 & 2.32323 & 0.4832 & 21.897 & 0.009 & 20.787 & 0.004 & 20.188 & 0.002 & 19.837 & 0.004 & 19.535 & 0.006 & 18.743 & 0.023 & 18.846 & 0.052 & 10.687 & 0.412 \\
\hline 1 & 0.02478 & 2.32432 & 0.4857 & 22.286 & 0.010 & 20.856 & 0.004 & 20.087 & 0.002 & 19.791 & 0.003 & 19.533 & 0.006 & 18.801 & 0.024 & 19.049 & 0.063 & 10.732 & 0.468 \\
\hline 1 & 0.01072 & 2.34266 & 0.4389 & 23.039 & 0.020 & 21.482 & 0.005 & 20.728 & 0.003 & 20.403 & 0.005 & 20.235 & 0.012 & 20.035 & 0.068 & 99.000 & 99.000 & 10.235 & 0.494 \\
\hline 1 & 0.01372 & 2.32647 & 0.3770 & 23.386 & 0.030 & 22.030 & 0.010 & 21.497 & 0.007 & 21.209 & 0.012 & 20.992 & 0.023 & 20.625 & 0.113 & 99.000 & 99.000 & 9.876 & 0.272 \\
\hline 1 & 0.02198 & 2.32837 & 0.4442 & 22.571 & 0.013 & 20.863 & 0.003 & 20.092 & 0.002 & 19.746 & 0.003 & 19.559 & 0.005 & 18.605 & 0.020 & 19.206 & 0.071 & 10.889 & 0.438 \\
\hline 1 & 0.00964 & 2.32917 & 0.3948 & 22.652 & 0.012 & 21.104 & 0.004 & 20.421 & 0.002 & 20.037 & 0.003 & 19.853 & 0.006 & 19.033 & 0.029 & 19.263 & 0.076 & 10.757 & 0.244 \\
\hline 2 & 0.01831 & 2.30579 & 0.6107 & 23.615 & 0.024 & 21.711 & 0.006 & 20.502 & 0.002 & 20.078 & 0.003 & 19.836 & 0.006 & 18.814 & 0.024 & 19.432 & 0.087 & 11.109 & 0.541 \\
\hline 2 & 0.02242 & 2.30766 & 0.6322 & 23.368 & 0.036 & 22.213 & 0.019 & 21.889 & 0.017 & 21.337 & 0.022 & 21.687 & 0.041 & 19.352 & 0.038 & 19.593 & 0.100 & 10.419 & 0.510 \\
\hline 2 & 0.01087 & 2.31278 & 0.6132 & 22.804 & 0.016 & 21.673 & 0.007 & 20.951 & 0.004 & 20.723 & 0.007 & 20.526 & 0.014 & 19.656 & 0.050 & 99.000 & 99.000 & 10.335 & 0.403 \\
\hline 2 & 0.01134 & 2.31443 & 0.6628 & 24.218 & 0.036 & 22.516 & 0.009 & 21.384 & 0.003 & 20.955 & 0.006 & 20.760 & 0.010 & 19.568 & 0.046 & 99.000 & 99.000 & 10.666 & 0.362 \\
\hline 2 & 0.01732 & 2.31986 & 0.6291 & 22.743 & 0.017 & 22.316 & 0.011 & 21.963 & 0.008 & 21.978 & 0.020 & 21.723 & 0.033 & 20.073 & 0.070 & 99.000 & 99.000 & 9.798 & 0.198 \\
\hline 2 & 0.01042 & 2.32136 & 0.6165 & 22.443 & 0.011 & 21.643 & 0.006 & 21.117 & 0.004 & 20.935 & 0.008 & 20.743 & 0.015 & 20.308 & 0.086 & 99.000 & 99.000 & 9.903 & 0.235 \\
\hline 2 & 0.02610 & 2.31993 & 0.5636 & 22.503 & 0.013 & 21.005 & 0.004 & 20.051 & 0.002 & 19.674 & 0.003 & 19.529 & 0.006 & 18.618 & 0.021 & 99.000 & 99.000 & 11.073 & 0.289 \\
\hline 2 & 0.01256 & 2.33595 & 0.6015 & 24.670 & 0.091 & 23.380 & 0.030 & 22.603 & 0.011 & 22.368 & 0.021 & 22.235 & 0.053 & 21.077 & 0.168 & 99.000 & 99.000 & 9.743 & 0.227 \\
\hline 2 & 0.01741 & 2.32800 & 0.6630 & 22.303 & 0.012 & 20.770 & 0.004 & 19.557 & 0.001 & 19.087 & 0.002 & 18.756 & 0.003 & 17.721 & 0.011 & 18.116 & 0.029 & 11.558 & 0.000 \\
\hline 2 & 0.01587 & 2.32853 & 0.6682 & 23.655 & 0.026 & 22.065 & 0.007 & 20.885 & 0.003 & 20.461 & 0.004 & 20.141 & 0.007 & 18.690 & 0.023 & 19.011 & 0.062 & 11.078 & 0.040 \\
\hline 2 & 0.01787 & 2.32850 & 0.6675 & 24.070 & 0.049 & 22.193 & 0.010 & 21.160 & 0.004 & 20.737 & 0.007 & 20.488 & 0.012 & 18.652 & 0.024 & 99.000 & 99.000 & 11.018 & 0.017 \\
\hline 2 & 0.00335 & 2.33072 & 0.6702 & 22.734 & 0.010 & 21.801 & 0.005 & 20.484 & 0.002 & 20.120 & 0.003 & 20.037 & 0.005 & 19.430 & 0.041 & 20.078 & 0.154 & 10.784 & 0.348 \\
\hline 2 & 0.02441 & 2.33236 & 0.5731 & 23.923 & 0.034 & 22.311 & 0.013 & 21.265 & 0.004 & 20.806 & 0.006 & 20.550 & 0.012 & 19.534 & 0.045 & 19.719 & 0.112 & 10.760 & 0.201 \\
\hline 2 & 0.02096 & 2.33169 & 0.6769 & 22.583 & 0.013 & 21.593 & 0.006 & 20.733 & 0.003 & 20.387 & 0.005 & 20.241 & 0.009 & 19.078 & 0.030 & 19.444 & 0.088 & 10.693 & 0.125 \\
\hline 2 & 0.04241 & 2.33564 & 0.6069 & 22.696 & 0.035 & 21.569 & 0.010 & 20.604 & 0.004 & 20.345 & 0.006 & 20.150 & 0.011 & 19.565 & 0.045 & 20.042 & 0.147 & 10.540 & 0.636 \\
\hline 2 & 0.02966 & 2.33760 & 0.6790 & 22.542 & 0.016 & 21.765 & 0.007 & 21.078 & 0.004 & 20.832 & 0.008 & 20.614 & 0.014 & 19.542 & 0.045 & 19.978 & 0.140 & 10.344 & 0.379 \\
\hline 2 & 0.02303 & 2.34174 & 0.6420 & 25.040 & 0.084 & 23.428 & 0.023 & 22.320 & 0.008 & 21.894 & 0.015 & 21.642 & 0.025 & 20.750 & 0.127 & 99.000 & 99.000 & 10.143 & 0.361 \\
\hline
\end{tabular}

Note. Column 1: Cluster ID in Table 1; Column 2 and 3: Right Ascension (R.A. J2000) and Declination (Dec. J2000) of member galaxy candidate (in degree); Column 4: photometric redshift of the galaxy; Column 5-18: magnitude and error in the $g, r, i, Z, y$, W1 and W2 bands. The value of 99.000 means no data; Column 19: $\operatorname{logarithm}$ of galaxy stellar mass, $\log \left(m_{\text {stellar }} / M_{\odot}\right)$; Column 20 : projected distance to cluster centre, in Mpc.

(This table is available in its entirety in a machine-readable form.) 

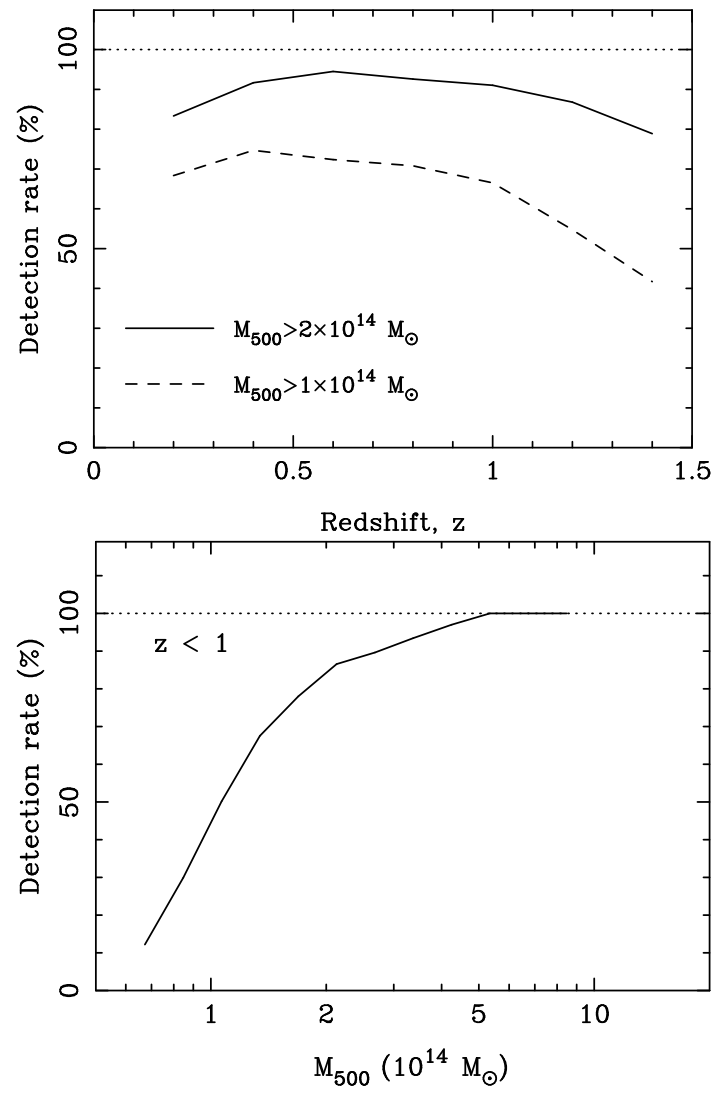

Figure 11. Detection rate of mock clusters as a function of redshift (upper) and cluster mass (lower) for completeness tests.

\subsection{Completeness estimate}

Completeness is one of necessary evaluations for an optical cluster catalogue. It can be estimated by two methods: the cross-matching with X-ray cluster catalogues and the Monte-Carlo simulations. The former is usually used for low redshift clusters (e.g. Oguri 2014; Wen et al. 2018). The latter uses mock clusters based on simulations (e.g. Koester et al. 2007; Wen et al. 2009; Hao et al. 2010; Wen et al. 2012; Rykoff et al. 2014; Oguri et al. 2018). Here, Monte-Carlo simulations are performed to estimate completeness of our cluster catalogue following our previous work (Wen et al. 2012).

In the HSC-SSP field, we generate a population of mock clusters with a mass function of Tinker et al. (2008) within a volume of $z<1.5$. The total stellar mass inside a cluster is derived following the cluster mass-richness relation

$\log M_{500}=0.96 \log \left(C \times \lambda_{500}\right)-1.29$,

where the factor $C$ is the ratio between the input and output richness due to the uncertainty of galaxy photometric redshift. The value of $C$ is to be determined so that the output richness follows the Eq. (14). For each mock cluster, the member galaxy population is obtained following the stellar mass function by van der Burg et al. (2018). The photometric redshift of each member galaxy is given as the cluster redshift $(z)$ plus an error, i.e. $z+z_{\text {err }}$, where $z_{\text {err }}$ is a Gaussian random number with a scatter of the redshift uncertainty shown in Fig. 1. The surface profile of member galaxies follows a two-dimensional NFW profile (Bartelmann 1996) with a concentration of $c_{500}=2$ (Wen \& Han 2018). The final mock member galaxies are obtained by consider-

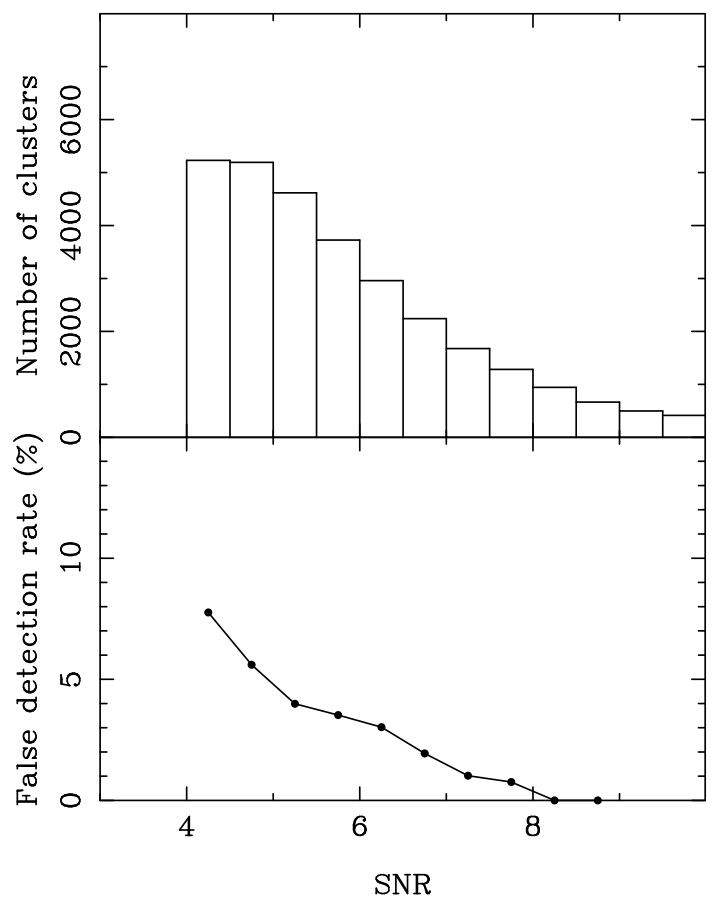

Figure 12. Distribution of cluster detection SNR (upper) and the false detection rate against SNR (lower).

ing the completeness of galaxies as functions of redshift and stellar mass shown in Fig. 3.

The completeness simulation is performed by two steps. First, we set $C=1$ to generate the mock clusters and their member galaxies. After adding the mock member galaxies into the observed HSC-SSP $\times$ unWISE data, we apply the procedure of cluster identification in Section 3.2 to identify mock clusters. The output richness is scaled to the input richness by $C=1 / 0.82$ (Wen et al. 2012). Secondly, we re-generate the mock member galaxies using the Eq. (15) with the new value of $C$ and add them into the HSC-SSP $\times$ unWISE data. Again, the procedure of cluster identification in Section 3.2 is applied to identify the mock clusters. The detection rate of mock clusters is regarded as a completeness estimate of our cluster catalogue, which is defined to be the ratio between the number of identified mock clusters and the total number of mock clusters. We show in the upper panel of Fig. 11 that the completeness slightly varies with redshift. For massive clusters of $M_{500}>2 \times 10^{14} M_{\odot}$, the completeness is $\gtrsim 90 \%$ up to $z \sim 1$ but decreases to $\sim 80 \%$ at $z \sim 1.5$. The completeness is about $70 \%$ up to $z \sim 1$ for clusters of $M_{500}>1 \times 10^{14} M_{\odot}$. Figure 11 also shows the completeness increases with cluster mass at $z<1$, from $50 \%$ at $M_{500} \sim 1 \times 10^{14} M_{\odot}$ to $85 \%$ at $M_{500} \sim 2 \times 10^{14} M_{\odot}$ (lower panel).

\subsection{False detection rate}

The filament structures along the line of sight show some overdensity regions of galaxy stellar mass. Due to the large photometric redshift slice we adopt, the projection effect may result in false detection of galaxy clusters.

Following previous works (Goto et al. 2002; Hao et al. 2010; Wen et al. 2012), we estimate the false detection rate using a mock galaxy catalogue of the HSC-SSP $\times$ unWISE data, in which known identified clusters are erased. To generate a mock galaxy cata- 


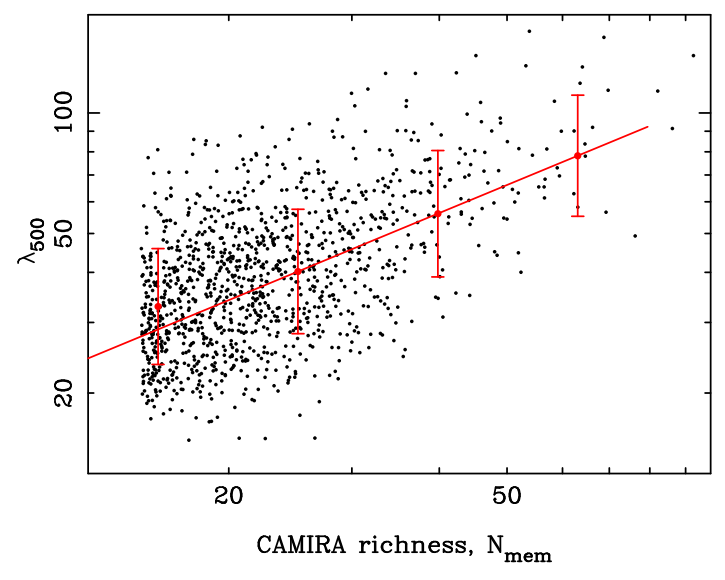

Figure 13. Correlation between the CAMIRA richness (Oguri et al. 2018) and the richness of this work. The red dots and error bars are the mean and scatter within each bin. The solid is the best fit of the correlation.

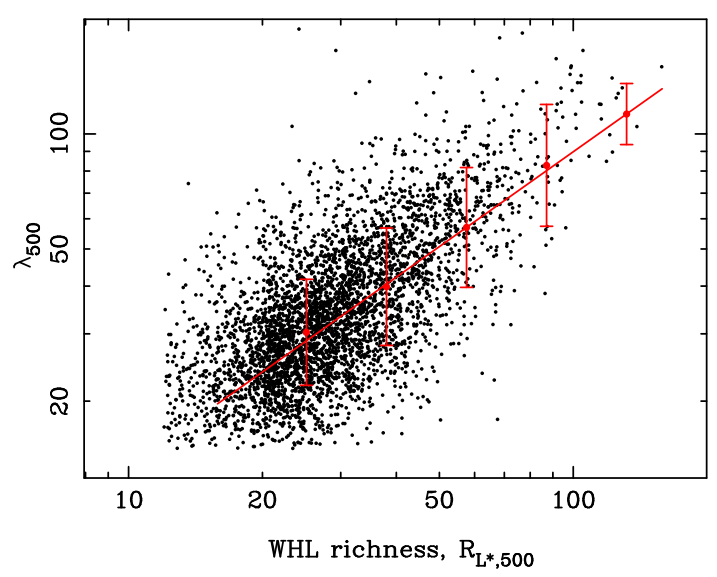

Figure 14. Similar to Fig. 13 but for the comparison with the WHL richness (Wen \& Han 2015).

logue, we first remove the member galaxy candidates within $r_{500}$ of clusters in this work and in Wen \& Han (2015) from the HSCSSP $\times$ unWISE galaxy catalogue. We then randomly shuffle the photometric redshifts of the rest galaxies, but keep the positions of galaxies on the sky unchanged. The mock catalogue should contain as few real clusters as possible, but have the same distribution of galaxies on the sky as the original catalogue. After these steps, we apply the procedures of cluster identification in Section 3.2 with the richness threshold of $\lambda_{500} \geq 15$. We adopt the detection threshold of SNR $\geq 4$ for this test. Since no real clusters are present in the mock galaxy catalogue, any detected "clusters" are regarded as false detections due to the projection effect. The false detection rate is defined as being the ratio between the number of false detections from the mock galaxy data and the total number of identified clusters from the real HSC-SSP $\times$ unWISE data.

We obtain ten mock catalogues of galaxies and get an average false detection rate, which decreases with cluster SNR from about $7 \%$ for clusters of $4<\mathrm{SNR}<5$ to about $4 \%$ for clusters of $5<$ SNR $<6$ and becomes zero for clusters of SNR $>8$, as shown in Fig. 12. We therefore set the threshold of SNR $\geq 5$ for cluster identification in this paper.

\subsection{Comparison with previous cluster catalogues}

In the HSC-SSP $\times$ unWISE region, many galaxy clusters have been recognized from multiwavelength data, such as optical cluster catalogues from the Cosmic Evolution Survey (COSMOS) data (Wen \& Han 2011), the SDSS data (Wen \& Han 2015) and the HSC-SSP data (Oguri et al. 2018), X-ray cluster catalogue from the XMM-Newton data (Adami et al. 2018) and SZ cluster catalogue from the ACT survey (Hilton et al. 2018). We cross-match with these catalogues to investigate how many known clusters in our cluster catalogue.

\subsubsection{HSC-SSP CAMIRA clusters}

Oguri et al. (2018) applied the Cluster finding Algorithm based on Multi-band Identification of Red-sequence gAlaxies (CAMIRA Oguri 2014) to the HSC-SSP S16A data covering $\sim 232 \mathrm{deg}^{2}$, and identified 1921 clusters in the redshift range of $0.1<z<1.1$. The CAMIRA algorithm calculates the likelihood of being redsequence galaxies as a function of redshift. The richness is defined to be the number of red member galaxies with a stellar mass greater than $10^{10.2} M_{\odot}$ for cluster candidates, and the identified clusters have a richness threshold of $N_{\mathrm{mem}} \geq 15$ and an equivalent mass of $M_{500} \gtrsim 1.2 \times 10^{14} M_{\odot}$ (Chiu et al. 2020). The BCG of each cluster was identified as the galaxy with the highest stellar mass near the richness peak and was taken as the cluster centre. The uncertainty of cluster redshift is better than 0.01 . Simulation shows that the cluster catalogue has a high $(>90 \%)$ completeness and purity. The HSC-SSP CAMIRA catalogue was obtained from the five-band (grizy) photometric data, and the colours are insensitive to redshift for galaxies of $z>1.4$ because the $4000 \AA$ break moves out of the $y$ band. Obviously, we extend the cluster sample to higher redshifts by combining the HSC-SSP data with the infrared data of the unWISE (see Fig. 8).

There are 1447 (i.e. 75\%) HSC-SSP CAMIRA clusters within a projected separation of $1.5 r_{500}$ and a redshift difference of $0.05(1+z)$ from the clusters in our catalogue. About $70 \%$ of the CAMIRA clusters with a richness of $N_{\text {mem }} \sim 20$ are matched with the clusters in Table 1 , and about $>90 \%$ clusters of $N_{\text {mem }} \geq 30$ are matched. The richness of CAMIRA clusters is related to our value (see Fig. 13) by a power law of

$\log \lambda_{500}=(0.72 \pm 0.03) \log N_{\text {mem }}+(0.59 \pm 0.04)$.

In other words, the threshold of CAMIRA richness of $N_{\text {mem }}=15$ is equivalent to our richness of $\lambda_{500} \sim 27$, suggesting that the CAMIRA catalogue has a larger mass threshold than our cluster catalogue.

\subsubsection{SDSS clusters}

Some of identified clusters from the HSC-SSP $\times$ unWISE data have been detected from the SDSS data (WHL, Wen et al. 2012). The richness is defined to be the total luminosity of member galaxies in units of $L^{*}$. Wen \& Han (2015) updated the WHL cluster catalogue with the spectroscopic redshifts of the SDSS and identified additional 25,419 new clusters at higher redshifts, so that the combined WHL catalogue contains 158,103 clusters.

In the HSC-SSP $\times$ unWISE region, there are 7464 WHL clusters and 4689 (i.e. $63 \%$ ) of them are matched with the clusters in Table 1 within a projected separation of $1.5 r_{500}$ and a redshift difference of $0.05(1+z)$, most of which have redshifts $z<0.65$. About $65 \%$ of these WHL clusters with a richness of $R_{L *, 500} \sim 30$ 


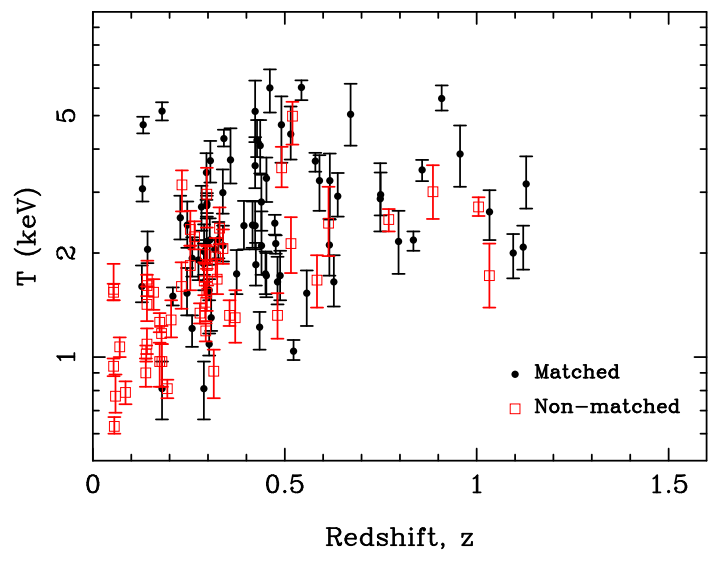

Figure 15. Matched and non-matched XXL clusters.

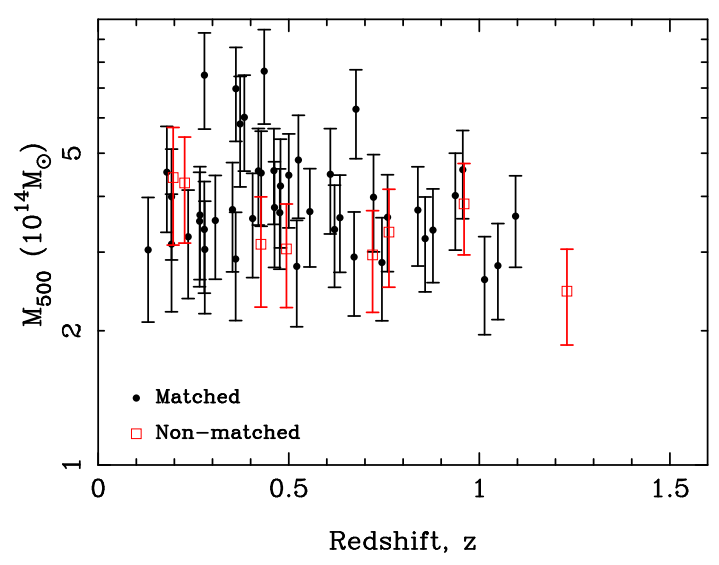

Figure 16. Matched and non-matched ACT clusters.

are matched and about $85 \%$ of the WHL clusters of $R_{L *, 500} \sim 50$ are matched. Fig. 14 shows the correlation between the richness of this work and the richness in the WHL catalogue. The best fit to a power law gives

$\log \lambda_{500}=(0.83 \pm 0.01) \log R_{L *, 500}+(0.26 \pm 0.02)$.

\subsubsection{X-ray clusters}

We compare our clusters with those identified from X-ray observations. A few deep X-ray surveys have been carried out in the HSC-SSP $\times$ unWISE coverage, including XMM-Large-Scale Structure (LSS) field survey (Clerc et al. 2014), XMM-COSMOS field survey (Hasinger et al. 2007) and XMM-XXL field survey (Pierre et al. 2016). The XMM-XXL is among the largest XMM program covering two extragalactic areas of $25 \mathrm{deg}^{2}$ each with a sensitivity of $\sim 5 \times 10^{-15} \mathrm{erg} \mathrm{s}^{-1}$ in the $0.5-2 \mathrm{keV}$ band for point sources. The XXL cluster catalogue contains 365 clusters at $z<1.2$ (Adami et al. 2018).

In the HSC-SSP $\times$ unWISE region, we get 122 XXL clusters with temperature data, of which 75 clusters are matched by our cluster catalogue. Fig. 15 shows the XXL clusters in the temperature-redshift plane. About $80 \%$ of XXL clusters of $T=2$ $4.5 \mathrm{keV}$ and about $90 \%$ of clusters of $T>4.5 \mathrm{keV}$ are found in the Table 1 .

\subsubsection{SZ clusters}

The SZ effect has the advantage to detect high-redshift clusters because of its redshift-independent brightness. The ACT survey has been carried out in the HSC-SSP $\times$ unWISE coverage to identify high-redshift SZ clusters (Hasselfield et al. 2013). The latest ACT cluster catalogue includes $182 \mathrm{SZ}$ clusters in the redshift range of $0.1<z<1.4$ from the $987.5 \mathrm{deg}^{2}$ celestial equator field (Hilton et al. 2018). There are 52 ACT clusters in the HSCSSP $\times$ unWISE region, of which $44(85 \%)$ clusters are matched by our cluster catalogue (Fig. 16), and eight non-matched clusters have a lower SNR or a lower richness than the threshold for cluster identification by our algorithm.

\subsubsection{Clusters in the COSMOS field}

The COSMOS is designed to study evolution of galaxies, active galactic nuclei and large scale structure over the redshift range of $0.5<z<6$ using deep imaging and spectroscopic observations (Scoville et al. 2007a), covering an area of $2 \mathrm{deg}^{2}$ at the wavelengths of near-ultraviolet (NUV; e.g. Zamojski et al. 2007), optical (e.g. Scoville et al. 2007b; Taniguchi et al. 2007), infrared (e.g. Sanders et al. 2007) and X-ray (e.g. Hasinger et al. 2007; Civano et al. 2016). Clusters/groups have been identified using spectroscopic data (e.g. Knobel et al. 2012), the 30band NUV-infrared photometric data (e.g. Zatloukal et al. 2007; Bellagamba et al. 2011; Wen \& Han 2011) and X-ray imaging data (e.g. Finoguenov et al. 2007). In our catalogue, there are 72 clusters in the COSMOS field, of which 68 clusters have been previously detected (Finoguenov et al. 2007; Zatloukal et al. 2007; Grove et al. 2009; Wen \& Han 2011; Wen et al. 2012; Söchting et al. 2012; Allevato et al. 2012; Balogh et al. 2014), and four clusters are newly identified at $z>0.8$. Such a high matched rate suggests that our clusters is fairly reliable, verifying the low false detection rate based on the Monte-Carlo simulation in Section 3.4.

\section{PROPERTIES OF MEMBER GALAXIES}

Using identified clusters in a wide redshift range from the HSCSSP $\times$ unWISE data, we study the evolution of BCG stellar mass and star formation.

\subsection{Stellar mass evolution of BCGs}

According to the hierarchical model of galaxy formation, the population of BCGs was formed at early universe and grew through the merging of satellite galaxies (De Lucia \& Blaizot 2007). However, no significant evolution of the BCG stellar mass is shown in optical selected clusters since $z \sim 1$ (Whiley et al. 2008), probably due to the selection effect because BCGs co-evolve with their host clusters. Lidman et al. (2012) found that the BCG stellar mass increases by a factor of $1.8 \pm 0.3$ from $z=0.9$ to $z=0.2$, but the growth is much slower than the prediction of the semi-analytic model in De Lucia \& Blaizot (2007). Bellstedt et al. (2016) argued that the growth of BCG stellar mass is consistent with the model. In addition, the BCG stellar mass is related to cluster mass. Zhang et al. (2016) found the scaling relation of $m_{\text {stellar }} \propto M_{200}^{0.24 \pm 0.08}(1+z)^{-0.19 \pm 0.34}$ using 106 X-ray selected cluster of $z<1.2$. Erfanianfar et al. (2019) found from 416 BCGs 

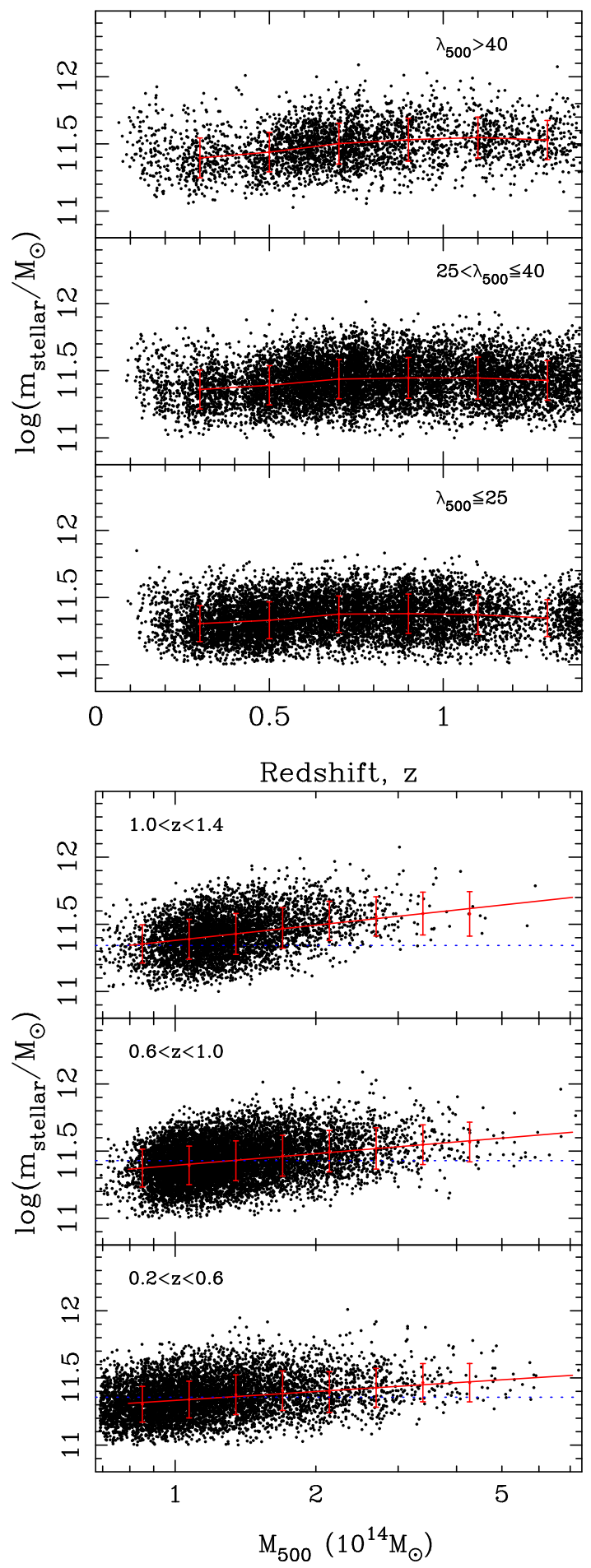

Figure 17. Upper: BCG stellar mass as a function of redshift within three richness bins. Lower: correlation between BCG stellar mass and cluster mass within three redshift bins. The red solid lines are the best fit to the data.

that the dependence of stellar masses on halo mass does not significantly change within $0.1<z<0.65$. The slope is $0.41 \pm 0.04$ within $0.1 \leq z \leq 0.3$ and $0.31 \pm 0.02$ within $0.3<z \leq 0.65$. Using 42 groups and clusters at $0.05<z<1.75$, DeMaio et al. (2020) found the scaling relation of $m_{\text {stellar }} \propto M_{500}^{0.48 \pm 0.06}$ for the BCG plus intracluster light. The normalization does not change from $z=0.1$ to $z=0.4$, but is $2.08 \pm 0.21$ times higher than that at $z=1.55$.

Using the large number of clusters in a wide redshift range, we can investigate the evolution of BCG stellar mass with redshift as well as the dependence on cluster mass. First, we divide our cluster sample into three richness bins and study the redshift evolution of BCG stellar mass. Within each richness bin, the BCG stellar mass has a scatter of 0.14 dex and does not evolve with redshift (upper panel of Fig. 17). Then, we divide our cluster sample into three redshift bins and study the dependence of BCG stellar mass on cluster mass. We find that the BCG stellar mass is positively related to cluster mass (lower panels of Fig. 17) by the relations

$$
\begin{array}{r}
\log m_{\text {stellar }}=(0.23 \pm 0.02) \log M_{500}+(11.33 \pm 0.01) \\
\text { for } 0.2<z<0.6 ; \\
\log m_{\text {stellar }}=(0.29 \pm 0.01) \log M_{500}+(11.39 \pm 0.01) \\
\text { for } 0.6<z<1.0 ; \\
\log m_{\text {stellar }}=(0.38 \pm 0.03) \log M_{500}+(11.38 \pm 0.02) \\
\text { for } 1.0<z<1.4,
\end{array}
$$

which implies no significant evolution with redshift but dependence on cluster environments. The slope we obtain is consistent with that of Zhang et al. (2016), but slightly lower than those in Erfanianfar et al. (2019) and DeMaio et al. (2020) and also that of 0.4-0.5 from the theoretical model (Whiley et al. 2008).

Because of the BCG-cluster co-evolution, the results shown in Fig. 17 do not mean that the BCG stellar mass is constant over cosmic time because cluster mass may evolve with redshift. The upper panel of Fig. 17 is obtained from the given range of cluster richness. According to the formula of halo merger rate (Fakhouri et al. 2010), the clusters are about three times more massive at $z=1$ than those at $z=0$. Using the Eq. (18) (adopting an average slope of 0.3 ), we find that the BCG stellar mass increases by a factor of 1.5 from the redshift of $z=1$ to $z=0$, consistent with the suggestions by Lidman et al. (2012) and Zhang et al. (2016), but lower than a factor of 3 by the semi-analytic model (De Lucia \& Blaizot 2007).

\subsection{Star formation in BCGs}

Optical and infrared data show that the stellar population in most BCGs is in agreement with a passive evolution. However, BCGs in some cool-core clusters have signatures of ongoing star formation (Crawford et al. 1999; Liu et al. 2012), though the fraction of starforming BCGs is often low.

The star formation rates of the BCGs can be obtained by fitting the 7-band photometry to the Fitting and Assessment of Synthetic Templates code (FAST; Kriek et al. 2009). We adopt the stellar population synthesis models from Bruzual \& Charlot (2003) and the initial mass function of Chabrier (2003), and also an exponentially declining star formation history. We calculate the fraction of BCGs with a star formation rate greater than $1.0 M_{\odot} \mathrm{yr}^{-1}$ for the clusters of different redshift bins and richness bins. As shown in the upper panel of Fig. 18, the fraction increases with redshift for all three richness bins, from about $8 \%$ at $z \sim 0.2$ to $20 \%$ at $z \sim 1.4$. In the lower panel of Fig. 18, we find that the fraction of star-forming BCGs does not significantly vary with cluster mass. Previously, Cerulo et al. (2019) found that a fraction of 9\% of BCGs are star forming at $z \sim 0.2$ based on the SDSS and WISE data. Webb et al. (2015) presented a $24 \mu \mathrm{m}$ study of 535 BCGs of $0.2<z<1.8$ from the Spitzer Adaptation of the Red-Sequence Cluster Survey. 

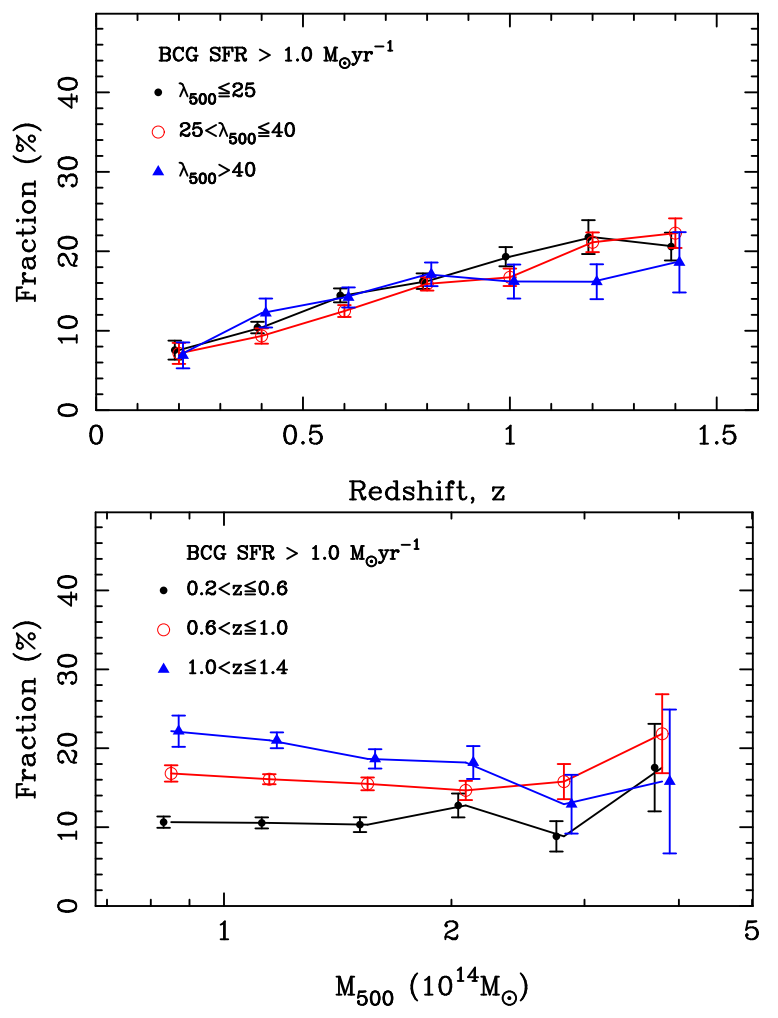

Figure 18. Fraction of star-forming BCGs as a function of redshift (upper) and cluster mass (lower).

They found that $\sim 20 \%$ of BCGs at $z>1$ have a high infrared luminosity of $L_{I R}>10^{12} L_{\odot}$ powered by star formation. Our results in Fig. 18 are consistent with these conclusions.

\section{SUMMARY}

We obtain a catalogue of photometric redshifts for 14.68 million HSC-SSP $\times$ unWISE galaxies. The photometric redshifts of galaxies are estimated in the colour space based on the nearest-neighbour algorithm. The redshift uncertainty is about 0.024 at redshifts $z<$ 0.7 and increases with redshift to about 0.11 at $z \sim 2$. Comparing to the public HSC-SSP redshifts, we improve the accuracy of photometric redshifts for galaxies within $1.4<z<2$. The stellar masses of galaxies are derived from the infrared magnitude and calibrated using the COSMOS data. The HSC-SSP $\times$ unWISE catalogue is $>90 \%$ complete at $z<0.5$ for galaxies with stellar masses $>10^{10.5} M_{\odot}$ and becomes $\sim 70 \%$ complete at $z \sim 1$.

From this large photometric redshift catalogue, we then identify 21,661 clusters in the redshift range of $0.1<z \lesssim 2$ with a detection signal-to-noise ratio $\geq 5$ and an equivalent mass $M_{500} \geq$ $0.7 \times 10^{14} M_{\odot}$. The uncertainty of cluster redshift is about 0.022 . Simulations show that the completeness is more than $90 \%$ for massive clusters of $M_{500}>2 \times 10^{14} M_{\odot}$ at $z<1$ and the false detection is less than $5 \%$. We study the stellar mass and star formation rate of the BCGs. We find no significant redshift evolution for the scaling relation between BCG stellar mass and cluster mass. The fraction of star-forming BCGs increases with redshift, but does not depend on cluster mass.

\section{ACKNOWLEDGEMENTS}

We thank the referee for valuable comments that helped to improve the paper. We are grateful to Dr. F. S. Liu for discussions. Dr. L. G. Hou and X. M. Meng help to figure out software. The authors are partially supported by the National Natural Science Foundation of China (Grant No. 11988101 and U1731127), the Key Research Program of the Chinese Academy of Sciences (Grant No. QYZDJSSW-SLH021) and the strategic Priority Research Program of Chinese Academy of Sciences (Grant No. XDB23010200), and the Open Project Program of the Key Laboratory of FAST, NAOC, Chinese Academy of Sciences. The HSC collaboration includes the astronomical communities of Japan and Taiwan, and Princeton University. The HSC instrumentation and software were developed by the National Astronomical Observatory of Japan (NAOJ), the Kavli Institute for the Physics and Mathematics of the Universe (Kavli IPMU), the University of Tokyo, the High Energy Accelerator Research Organization (KEK), the Academia Sinica Institute for Astronomy and Astrophysics in Taiwan (ASIAA), and Princeton University. Funding was contributed by the FIRST program from Japanese Cabinet Office, the Ministry of Education, Culture, Sports, Science and Technology (MEXT), the Japan Society for the Promotion of Science (JSPS), Japan Science and Technology Agency (JST), the Toray Science Foundation, NAOJ, Kavli IPMU, KEK, ASIAA, and Princeton University.

This paper has used software developed for the Large Synoptic Survey Telescope. We thank the LSST Project for making their code available as free software at http://dm.lsst.org

This paper is based [in part] on data collected at the Subaru Telescope and retrieved from the HSC data archive system, which is operated by Subaru Telescope and Astronomy Data Center at NAOJ. Data analysis was in part carried out with the cooperation of Center for Computational Astrophysics, NAOJ.

This publication has used data products from the Wide-field Infrared Survey Explorer, which is a joint project of the University of California, Los Angeles, and the Jet Propulsion Laboratory/California Institute of Technology, funded by the National Aeronautics and Space Administration.

\section{DATA AVAILABILITY}

The data of 14.68 million HSC-SSP $\times$ unWISE galaxies (7-band magnitudes, photometric redshifts and stellar masses), 21,661 identified clusters and their member galaxy candidates are publicly available at http://zmtt.bao.ac.cn/galaxy_clusters/.

\section{REFERENCES}

Abell G. O., 1958, ApJS, 3, 211

Abell G. O., Corwin Jr. H. G., Olowin R. P., 1989, ApJS, 70, 1

Abolfathi B., et al., 2018, ApJS, 235, 42

Adami C., et al., 2018, A\&A, 620, A5

Aihara H., et al., 2018, PASJ, 70, S4

Aihara H., et al., 2019, PASJ, p. 106

Allen S. W., Rapetti D. A., Schmidt R. W., Ebeling H., Morris R. G., Fabian A. C., 2008, MNRAS, 383, 879

Allen S. W., Evrard A. E., Mantz A. B., 2011, ARA\&A, 49, 409

Allevato V., et al., 2012, ApJ, 758, 47

Balogh M. L., et al., 2014, MNRAS, 443, 2679

Banerjee P., Szabo T., Pierpaoli E., Franco G., Ortiz M., Oramas A., Tornello B., 2018, New Astron., 58, 61

Bartelmann M., 1996, A\&A, 313, 697 
Beck R., Dobos L., Budavári T., Szalay A. S., Csabai I., 2016, MNRAS, 460,1371

Bell E. F., de Jong R. S., 2001, ApJ, 550, 212

Bellagamba F., Maturi M., Hamana T., Meneghetti M., Miyazaki S., Moscardini L., 2011, MNRAS, 413, 1145

Bellstedt S., et al., 2016, MNRAS, 460, 2862

Bernardi M., Shankar F., Hyde J. B., Mei S., Marulli F., Sheth R. K., 2010, MNRAS, 404, 2087

Bilicki M., et al., 2016, ApJS, 225, 5

Binggeli B., Tammann G. A., Sandage A., 1987, AJ, 94, 251

Böhringer H., Chon G., Collins C. A., Guzzo L., Nowak N., Bobrovskyi S., 2013, A\&A, 555, A30

Böhringer H., Chon G., Retzlaff J., Trümper J., Meisenheimer K., Schartel N., 2017, AJ, 153, 220

Bolzonella M., Miralles J. M., Pelló R., 2000, A\&A, 363, 476

Bradshaw E. J., et al., 2013, MNRAS, 433, 194

Brammer G. B., van Dokkum P. G., Coppi P., 2008, ApJ, 686, 1503

Brodwin M., et al., 2012, ApJ, 753, 162

Bruzual G., Charlot S., 2003, MNRAS, 344, 1000

Butcher H., Oemler Jr. A., 1978, ApJ, 219, 18

Butcher H., Oemler Jr. A., 1984, ApJ, 285, 426

Carrasco Kind M., Brunner R. J., 2014, MNRAS, 438, 3409

Cerulo P., Orellana G. A., Covone G., 2019, MNRAS, 487, 3759

Chabrier G., 2003, PASP, 115, 763

Chiu I. N., Umetsu K., Murata R., Medezinski E., Oguri M., 2020, MNRAS, 495,428

Civano F., et al., 2016, ApJ, 819, 62

Clerc N., et al., 2014, MNRAS, 444, 2723

Cluver M. E., et al., 2014, ApJ, 782, 90

Colberg J. M., White S. D. M., Jenkins A., Pearce F. R., 1999, MNRAS, 308,593

Collister A. A., Lahav O., 2004, PASP, 116, 345

Cool R. J., et al., 2013, ApJ, 767, 118

Cooper M. C., et al., 2011, ApJS, 193, 14

Crawford C. S., Allen S. W., Ebeling H., Edge A. C., Fabian A. C., 1999, MNRAS, 306, 857

Cunha C. E., Lima M., Oyaizu H., Frieman J., Lin H., 2009, MNRAS, 396,2379

Cutri R. M., et al. 2013, VizieR Online Data Catalog, 2328

De Lucia G., Blaizot J., 2007, MNRAS, 375, 2

DeMaio T., et al., 2020, MNRAS, 491, 3751

Drinkwater M. J., et al., 2010, MNRAS, 401, 1429

Drory N., Bender R., Hopp U., 2004, ApJ, 616, L103

Eisenhardt P. R. M., et al., 2008, ApJ, 684, 905

Erfanianfar G., et al., 2013, ApJ, 765, 117

Erfanianfar G., et al., 2019, A\&A, 631, A175

Fakhouri O., Ma C.-P., Boylan-Kolchin M., 2010, MNRAS, 406, 2267

Finoguenov A., et al., 2007, ApJS, 172, 182

Garilli B., et al., 2014, A\&A, 562, A23

Girardi L., Bressan A., Chiosi C., Bertelli G., Nasi E., 1996, A\&AS, 117,113

Gladders M. D., Yee H. K. C., 2005, ApJS, 157, 1

Gonzalez A. H., et al., 2019, ApJS, 240, 33

Goto T., et al., 2002, AJ, 123, 1807

Grove L. F., Benoist C., Martel F., 2009, A\&A, 494, 845

Hao J., et al., 2010, ApJS, 191, 254

Hasinger G., et al., 2007, ApJS, 172, 29

Hasselfield M., et al., 2013, J. Cosmology Astropart. Phys., 7, 008

Hilton M., et al., 2018, ApJS, 235, 20

Hoyle B., Jimenez R., Verde L., 2011, Phys. Rev. D, 83, 103502

Hsieh B. C., Yee H. K. C., 2014, ApJ, 792, 102

Ilbert O., et al., 2006, A\&A, 457, 841

Jarrett T. H., et al., 2011, ApJ, 735, 112

Jee M. J., et al., 2009, ApJ, 704, 672

Kale R., Venturi T., Cassano R., Giacintucci S., Bardelli S., Dallacasa D., Zucca E., 2015, A\&A, 581, A23

Kashino D., et al., 2019, ApJS, 241, 10

Knobel C., et al., 2012, ApJ, 753, 121
Koester B. P., et al., 2007, ApJ, 660, 239

Kriek M., van Dokkum P. G., Labbé I., Franx M., Illingworth G. D., Marchesini D., Quadri R. F., 2009, ApJ, 700, 221

Laigle C., et al., 2016, ApJS, 224, 24

Laird E. S., et al., 2009, ApJS, 180, 102

Lang D., 2014, AJ, 147, 108

Le Fèvre O., et al., 2013, A\&A, 559, A14

Li H.-N., Wu H., Cao C., Zhu Y.-N., 2007, AJ, 134, 1315

Lidman C., et al., 2012, MNRAS, 427, 550

Lilly S. J., et al., 2009, ApJS, 184, 218

Lima M., Cunha C. E., Oyaizu H., Frieman J., Lin H., Sheldon E. S., 2008, MNRAS, 390, 118

Lin Y.-T., et al., 2017, ApJ, 851, 139

Liske J., et al., 2015, MNRAS, 452, 2087

Liu F. S., Mao S., Meng X. M., 2012, MNRAS, 423, 422

Mainzer A., et al., 2014, ApJ, 792, 30

Mancone C. L., Gonzalez A. H., Brodwin M., Stanford S. A., Eisenhardt P. R. M., Stern D., Jones C., 2010, ApJ, 720, 284

Mantz A., Allen S. W., Ebeling H., Rapetti D., Drlica-Wagner A., 2010, MNRAS, 406, 1773

McLure R. J., et al., 2013, MNRAS, 428, 1088

Momcheva I. G., et al., 2016, ApJS, 225, 27

Nishizawa A. J., Hsieh B.-C., Tanaka M., Takata T., 2020, arXiv e-prints, p. arXiv:2003.01511

Oguri M., 2014, MNRAS, 444, 147

Oguri M., et al., 2018, PASJ, 70, S20

Pacaud F., et al., 2016, A\&A, 592, A2

Papovich C., et al., 2010, ApJ, 716, 1503

Peebles P. J. E., 1980, The large-scale structure of the universe

Pierre M., et al., 2004, J. Cosmology Astropart. Phys., 9, 011

Pierre M., et al., 2016, A\&A, 592, A1

Piffaretti R., Arnaud M., Pratt G. W., Pointecouteau E., Melin J.-B., 2011, A\&A, 534, A109

Planck Collaboration et al., 2016, A\&A, 594, A27

Prakash A., et al., 2016, ApJS, 224, 34

Reichardt C. L., et al., 2013, ApJ, 763, 127

Rigopoulou D., et al., 2006, ApJ, 648, 81

Rood H. J., 1969, ApJ, 158, 657

Rykoff E. S., et al., 2014, ApJ, 785, 104

Sanders D. B., et al., 2007, ApJS, 172, 86

Schlafly E. F., Meisner A. M., Green G. M., 2019, ApJS, 240, 30

Scoville N., et al., 2007a, ApJS, 172, 1

Scoville N., et al., 2007b, ApJS, 172, 38

Shu Y., Koposov S. E., Evans N. W., Belokurov V., McMahon R. G., Auger M. W., Lemon C. A., 2019, MNRAS, 489, 4741

Silverman J. D., et al., 2015, ApJS, 220, 12

Skelton R. E., et al., 2014, ApJS, 214, 24

Smith C. M. A., Gear W. K., Smith M. W. L., Papageorgiou A., Eales S. A., 2019, MNRAS, 486, 4304

Söchting I. K., Coldwell G. V., Clowes R. G., Campusano L. E., Graham M. J., 2012, MNRAS, 423, 2436

Stott J. P., Edge A. C., Smith G. P., Swinbank A. M., Ebeling H., 2008, MNRAS, 384, 1502

Szabo T., Pierpaoli E., Dong F., Pipino A., Gunn J., 2011, ApJ, 736, 21

Tanaka M., 2015, ApJ, 801, 20

Tanaka M., et al., 2018, PASJ, 70, S9

Taniguchi Y., et al., 2007, ApJS, 172, 9

Tarrío P., Zarattini S., 2020, arXiv e-prints, p. arXiv:2005.06489

Tinker J., Kravtsov A. V., Klypin A., Abazajian K., Warren M., Yepes G., Gottlöber S., Holz D. E., 2008, ApJ, 688, 709

Tozzi P., et al., 2015, ApJ, 799, 93

Vikhlinin A., et al., 2009, ApJ, 692, 1060

Wang T., et al., 2016, ApJ, 828, 56

Webb T. M. A., et al., 2015, ApJ, 814, 96

Wen Z. L., Han J. L., 2011, ApJ, 734, 68

Wen Z. L., Han J. L., 2015, ApJ, 807, 178

Wen Z. L., Han J. L., 2018, MNRAS, 481, 4158

Wen Z. L., Han J. L., Liu F. S., 2009, ApJS, 183, 197 


\section{Wen \& Han}

Wen Z. L., Han J. L., Liu F. S., 2012, ApJS, 199, 34

Wen X.-Q., Wu H., Zhu Y.-N., Lam M. I., Wu C.-J., Wicker J., Zhao Y.-H., 2013, MNRAS, 433, 2946

Wen Z. L., Han J. L., Yang F., 2018, MNRAS, 475, 343

Westera P., Lejeune T., Buser R., Cuisinier F., Bruzual G., 2002, A\&A, 381,524

Whiley I. M., et al., 2008, MNRAS, 387, 1253

Willis J. P., Ramos-Ceja M. E., Muzzin A., Pacaud F., Yee H. K. C., Wilson G., 2018, MNRAS, 477, 5517

Wright E. L., et al., 2010, AJ, 140, 1868

Zamojski M. A., et al., 2007, ApJS, 172, 468

Zatloukal M., Röser H.-J., Wolf C., Hippelein H., Falter S., 2007, A\&A, 474, L5

Zhang Y., et al., 2016, ApJ, 816, 98

Zou H., Gao J., Zhou X., Kong X., 2019, ApJS, 242, 8

van Breukelen C., et al., 2006, MNRAS, 373, L26

van der Burg R. F. J., McGee S., Aussel H., Dahle H., Arnaud M., Pratt G. W., Muzzin A., 2018, A\&A, 618, A140 